OPEN ACCESS

Edited by:

Oliver Reiser,

University of Regensburg, Germany

Reviewed by:

Federica Valentini,

Università di Roma Tor Vergata, Italy

Till Opatz,

Johannes Gutenberg University

Mainz, Germany

*Correspondence:

Guigen Li

guigen.li@ttu.edu

Bo Jiang

jiangchem@jsnu.edu.cn

Specialty section

This article was submitted to

Green and Sustainable Chemistry,

a section of the journal

Frontiers in Chemistry

Received: 12 September 2018 Accepted: 19 November 2018 Published: 04 December 2018

Citation:

Li H, Hao W-J, Li G, Tu S-J and Jiang B (2018) Fe(III)-Catalyzed

Bicyclization of Yne-Allenones With

Indoles for the Atom-Economic

Synthesis of 3-Indolyl Cyclobutarenes.

Front. Chem. 6:599.

doi: 10.3389/fchem.2018.00599

\section{Fe(III)-Catalyzed Bicyclization of Yne-Allenones With Indoles for the Atom-Economic Synthesis of 3-Indolyl Cyclobutarenes}

\author{
Heng $\mathrm{Li}^{1}$, Wen-Juan Hao ${ }^{1}$, Guigen $\mathrm{Li}^{2,3 *}$, Shu-Jiang $\mathrm{Tu}^{1}$ and Bo Jiang ${ }^{1 *}$ \\ ${ }^{1}$ School of Chemistry and Materials Science, Jiangsu Key Laboratory of Green Synthetic Chemistry for Functional Materials, \\ Jiangsu Normal University, Xuzhou, China, ${ }^{2}$ Institute of Chemistry and BioMedical Sciences, Collaborative Innovation Center \\ of Chemistry for Life Sciences, Nanjing University, Nanjing, China, ${ }^{3}$ Department of Chemistry and Biochemistry, Texas Tech \\ University, Lubbock, TX, United States
}

A new Fe(III)-catalyzed bicyclization reaction of yne-allenones with indoles has been established, enabling the direct construction of cyclobuta[a]naphthalen-4-ols with an all-carbon quaternary center in good to excellent yields. This reaction was performed by using low-cost $\mathrm{FeCl}_{3}$ as the catalyst and $\mathrm{EtOH}$ as the environmentally benign solvent, providing a green protocol for constructing the cyclobutarene framework with a high degree of atom economy and functional group compatibility. The reaction mechanism was proposed to proceed through a [2 + 2] cycloaddition/1,6-conjugate addition cascade.

Keywords: Fe(III)-catalysis, bicyclization, 1,6-addition, yne-allenones, cyclobutarenes

\section{INTRODUCTION}

Development of practical and sustainable synthetic methods for the rapid construction of valuable cyclic target molecules, along with minimum environmental impacts, represents an endeavor of utmost importance in both academia, and industry (Anastas and Warner, 1998; Bruckmann et al., 2008; Martins et al., 2009; Jiang et al., 2010; Huang et al., 2018a). In this context, chemical transformations following the principles of atom-economy are generally believed to be green since such reactions enable different molecular fragments into integrated cyclic frameworks by recombining chemical bonds with maximum atom utilization and minimum generation of the chemical waste (Trost, 1995, 2002; Trost et al., 2003; Banert and Plefka, 2011; Kotha et al., 2013). The key to realize this goal is to implement reaction cascades, which allow the direct formation of multiple chemical bonds in a one-pot operation and can lead to a remarkable increase in resource efficiency for the overall process (Barluenga et al., 2009; Fuerstner, 2009; Tietze et al., 2009; Jones et al., 2010; Wang et al., 2015; Sugimoto and Matsuya, 2017; Zhang et al., 2017). Specifically, bicyclization cascades have emerged as an important platform for the synthesis of bioactive small-molecule libraries for their SAR studies (Dömling et al., 2012; Brauch et al., 2013; Vlaar et al., 2013; Koopmanschap et al., 2014; Rotstein et al., 2014; Huang et al., 2018b). Due to their annulation efficiency, economic and environmental aspects, and ease of operation as well as diminished waste disposals (Jia et al., 2014; Su et al., 2014; Tian et al., 2015; Chen et al., 2017; Huang et al., 2017; Liu et al., 2017b,c; Wang L. et al., 2017). In view of the environmental awareness of the chemical community, the combination of the presented bicyclization strategy and the use of environmentally benign solvents will furnish the transformations under avoidance of potential 
pollutants (Bihani et al., 2013; Wang J.-Y. et al., 2017; Sha et al., 2018a). Nevertheless, the design and development of environmentally compatible bicyclization cascades without generation of toxic waste and by-products holds considerable challenges.

Cyclobutarenes are a class of structurally unique bicarbocyclic molecules which show a wide spectrum of biological activity (Christophe et al., 1998; Sadana et al., 2003). Due to the thermodynamic stability associated with the aromatic system and the kinetic reactivity of the strained cyclobutene ring (Cava and Napier, 1956; Mehta and Kotha, 2001), these molecules behave as reliable and synthetically useful feedstocks (Christophe et al., 1998) and have been extensively applied in natural product syntheses (Funk and Vollhardt, 1977, 1979; Grieco et al., 1980; Taber et al., 1987; Nemoto et al., 1995; Michellys et al., 2001). With these contributions in mind, great efforts to establish synthetic protocols for cyclobutarene synthesis have been developed which include 1,4-elimination-cycloaddition of functionalized arenes (Gray et al., 1978; Schirch et al., 1979; Sekine et al., 1979; Lenihan and Shechter, 1994, 1998; Chou et al., 1995), Parham cyclization (Bradsher and Hunt, 1981; Buchwald et al., 1987a,b; Beak and Selling, 1989; Aidhen and Ahuja, 1992), photo-induced cycloadditions (Parham et al., 1976; Kaneko and Naito, 1979; Neckers and Wagenaar, 1981; Kaneko et al., 1982; Kanao et al., 1983; Sato et al., 1987; Hoffmann and Pete, 1996), thermal extrusion reactions (Toda et al., 1988; D’Andrea et al., 1990; Hickman et al., 1991; Shimada et al., 1993; Andersen et al., 1996; Craig et al., 1998), intramolecular addition of carbanions to benzynes (Bunnett and Skorcz, 1962; Krohn et al., 1978; Gowland and Durst, 1979), $[2+2+2]$ cycloadditions of 1,5-hexadiyne (Peter and Vollhardt, 1977, 1984; Funk and Vollhardt, 1980; McNichols and Stang, 1992), ring expansion of cycloproparenes (Birch et al., 1964; Iskander and Stansfield, 1965; Buckland et al., 1987; Kagabu and Saito, 1988; Müller et al., 1989), and [2 + 2] cycloadditions of allene precursors (Inanaga et al., 1992; Ezcurra and Moore, 1993; Toda et al., 1994) and other methods (Markgraf et al., 1969; Garratt and Nicolaides, 1972, 1974; Bilyard et al., 1979; Warrener et al., 1993). However, these methods encounter some drawbacks such as high temperatures $\left(400^{\circ} \mathrm{C}-800^{\circ} \mathrm{C}\right)$, strong bases $\left(n\right.$-BuLi and $\left.\mathrm{NaNH}_{2}\right)$, multiple steps, or a narrow substrate range. Moreover, indole derivatives stand for another important class of heterocyclic compounds present in a myriad of bioactive substances and natural products. Therefore, the development of general and sustainable entries toward cyclobutarene-indole pairs in atom- and poteconomic manner is of potential significance. Recently, we reported the combination of $[2+2]$ cycloaddition with 1,4-radical addition reaction by treating yne-allenones with aryldiazonium salts and DABCO-bis(sulfur dioxide) (DABSO), affording functional cyclobuta $[a]$ naphthalen-4-ols (Scheme 1a, Liu et al., 2017a). Subsequently, we developed a $\mathrm{BF}_{3} \bullet \mathrm{Et}_{2} \mathrm{O}-$ catalyzed double $[2+2]$ cycloaddition relay between yneallenones and unactivated alkenes, enabling $\mathrm{C}-\mathrm{C}$ triple bond cleavage to access phenanthren-9-ols (Scheme 1b, Li et al., 2018a). To continue our efforts in this project (Liu et al., 2017a; Wang J.-Y. et al., 2017; Li et al., 2018a,b; Sha et al., 2018b; Wang et al., 2018), we attempted to employ indoles 2 to be subjected with the reaction of yne-allenones 3 under our previous conditions ( $\mathrm{Li}$ et al., 2018a) to assemble naphtho[1,2a]carbazol-5-ols 4 (Scheme 1d), owing to indoles with $\mathrm{C} 2$ and $\mathrm{C} 3$ reactive sites could acted as $\mathrm{C}_{2}$ synthons for the synthesis of fused indoles (Haibach et al., 2011; Li et al., 2015; Liu et al., 2016; Ozaki et al., 2017). Unexpectedly, a double $[2+2]$ cycloaddition relay did not occur. Instead, the reaction involved another $[2+2]$ cycloaddition /1,6-addtition cascade to furnish 3-indolyl substituted cyclobuta[a]naphthalen4-ols 3 by suitably adjusting the catalysts and solvents (Scheme 1c). Notably, the current green protocol represents an atom-economic and eco-friendly entry to structurally unique cyclobutarene-indole pairs through the combination of $[2+$ 2] cycloaddition with 1,6-conjugate addition by $\mathrm{FeCl}_{3}$ as a low-cost catalyst and EtOH as an environmentally benign solvent. Herein, we elaborate this attractive and benign transformation.

\section{RESULTS AND DISCUSSION}

At the beginning of our studies, yne-allenone $\mathbf{1 a}$ and $\mathrm{N}$ methylindole (2a) were chosen as the model substrates to explore the feasibility of double $[2+2]$ cycloaddition relay reaction with our previous conditions (Table 1, entry 1). Instead of the expected naphtho[1,2-a]carbazol-5-ol 4a, 3-indolyl substituted cyclobuta $[a]$ naphthalen- 4 -ol 3 a was obtained in $60 \%$ yield. The following screening of solvents, such as $\mathrm{N}, \mathrm{N}$-dimethylformamide (DMF), 1,4-dioxane, tetrahydrofuran (THF), $\mathrm{MeOH}$, and $\mathrm{EtOH}$, showed that use of DMF and 1,4-dioxane as reaction media completely suppressed the reaction process (entries 2-3) whereas the latter three all made the transformations work more efficiently (entries 4-6). Among these, EtOH proved to be the best choice, providing the product $\mathbf{3 a}$ with the highest yield of $76 \%$ (entry 6). Increasing the component ratio to $1: 2$ is not beneficial for this transformation as a lower conversion was observed (63\%, entry 7$)$. In contrast, fine-tuning the component ratio to $1: 1.2$ could improve the reaction efficiency, resulting in a higher yield of $\mathbf{3 a}(81 \%$, entry 8$)$. As the next optimization step, we conducted the screening of a variety of Lewis acid catalysts, such as $\mathrm{ZnCl}_{2}, \mathrm{Y}(\mathrm{OTf})_{3}$ and $\mathrm{FeCl}_{3}$ that are often employed in the catalytic transformations, for this cyclization-addition cascade by using $\mathrm{EtOH}$ as the reaction media. The former two led to remarkably lower conversions (entries 9-10). Delightingly, the latter one showed the best catalytic performance in this transformation, delivering higher yield of $3 \mathbf{a}$ as compared with $\mathrm{BF}_{3} \bullet \mathrm{Et}_{2} \mathrm{O}$ (85\%, entry 11 vs. $8)$. It is found that the reaction efficiency was proven to display an important dependence on the loading of the $\mathrm{Fe}$ catalyst. An increase in the $\mathrm{FeCl}_{3}$ loading had a detrimental impact on the reaction yield (entry 12) whereas reducing the catalytic amount of $\mathrm{FeCl}_{3}$ to $10 \mathrm{~mol} \%$ could accelerate the conversion into $3 \mathrm{a}$ in an increased the yield to $88 \%$. When the reaction temperature was elevated to $70^{\circ} \mathrm{C}$, the reaction process was inhibited in some extent (entry 14). On the contrary, decreasing the reaction temperature to $30^{\circ} \mathrm{C}$ facilitated 


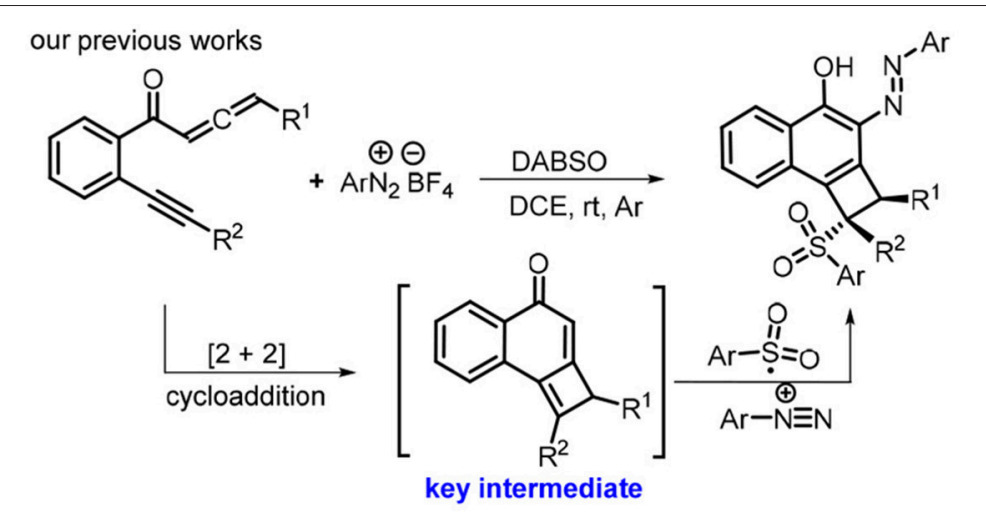

(a)

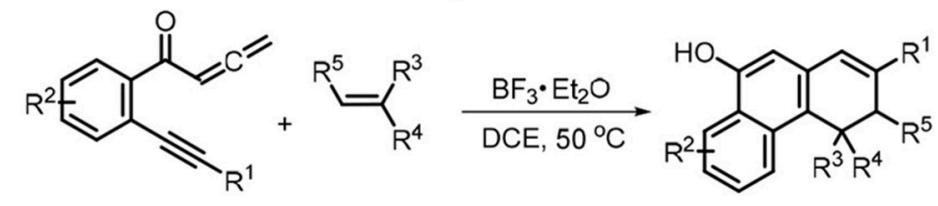

(b)
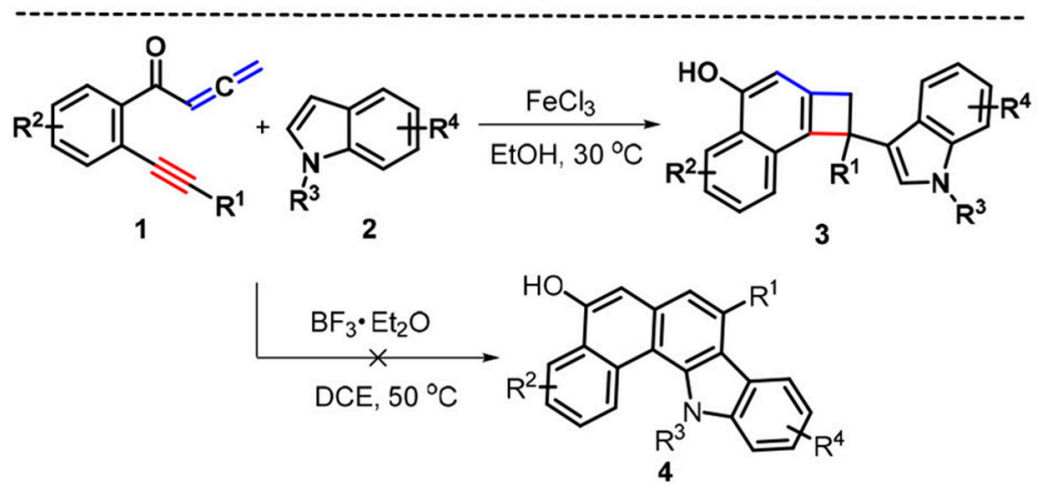

SCHEME 1 | Profiles for [2 + 2] cycloaddition of yne-allenones.

the current transformation and gave a higher yield of $90 \%$ (entry 15).

With these optimal conditions in hand (Table 1, entry 15), we set out to examine the scope of this Fe-catalyzed [2 + 2] cycloaddition /1,6-addition cascade by using a variety of yne-allenones and indoles. As depicted in Scheme 2, Nmethylindole (2a) was first selected to evaluate the influence of substituents $\left(\mathrm{R}^{1}\right)$ in the arylalkynyl moiety of yne-allenone 1. Both electron-poor and electron-rich groups at different positions of the arylalkynyl moiety $\left(\mathrm{R}^{1}\right)$ can all tolerate this catalytic system, efficiently accessing the corresponding products 3b-3j in 75-98\% yields. Diverse substituents, such as fluoro (1b), chloro (1c and 1d), bromo (1e), methyl (1f and 1g), methoxy $(\mathrm{PMP}=p$-methoxyphenyl, 1h), ethyl(1i), $t$-butyl $(\mathbf{1 j})$ were suitable for this transformation. The presence of soft electronwithdrawing substituents (chloro, 1c and bromo, 1e) at the para-positions seemed to result in higher reactivity than that of electron-donating counterparts (3c and $\mathbf{3 e}$ vs. $\mathbf{3} \mathbf{f}$ and $\mathbf{3 h}-\mathbf{3 j}$ ). Moreover, a sterically encumbered 1-naphthyl (1-Np) analog $\mathbf{1 k}$ was an effective candidate, which proceeded through a similar cyclization-addition process to give the corresponding product 3k in $77 \%$ yield. Besides, 2-thienyl counterpart $\mathbf{1 1}$ still showed high reactivity, delivering 2 -thienyl product 31 in $91 \%$ yield. Next, we placed different functional groups $\left(\mathrm{R}^{2}\right)$ including methoxy, methyl, and fluoro into the $\mathrm{C} 4$ or $\mathrm{C} 5$ position of the internal arene ring of substrates $\mathbf{1}$ and explored the synthetic utility of these substrates. Satisfyingly, all those substituents $(\mathbf{1} \mathbf{m}-\mathbf{1} \mathbf{u})$ would be compatible in the present reaction protocol, and the corresponding functionalized cyclobuta[a]naphthalen-4-ols 3m$3 \mathbf{u}$ in $72-92 \%$ yields were produced. Interestingly, the pyridinetethered yne-allenone $\mathbf{1 v}$ could be successfully converted into cyclobutarene product $3 \mathbf{v}$ in $68 \%$ yield.

Next, the scope with respect to indoles components was evaluated. As anticipated, the different substituents including methoxy (2b), chloro (1c and 1d), bromo (1e), methyl (1f and $\mathbf{1 g})$, located at different positions of the indole ring would be accommodated, confirming the reaction efficiency, as the cyclobuta[a]naphthalen-4-ol products $3 \mathbf{w}-\mathbf{3 b}$ b were offered in $76-92 \%$ yields. Finally, the free indole turned out to be a suitable reaction partner, leading to the formation of products $3 \mathrm{cc}$ and 3dd in 78 and $81 \%$ yields, respectively. Products 3 were fully characterized by their NMR and HR-MS spectral analysis. In the case of product $\mathbf{3 a}$, its structure was further confirmed by X-ray crystallography (Figure 1). 
TABLE 1 | Optimization of Reaction Conditions ${ }^{[a]}$.

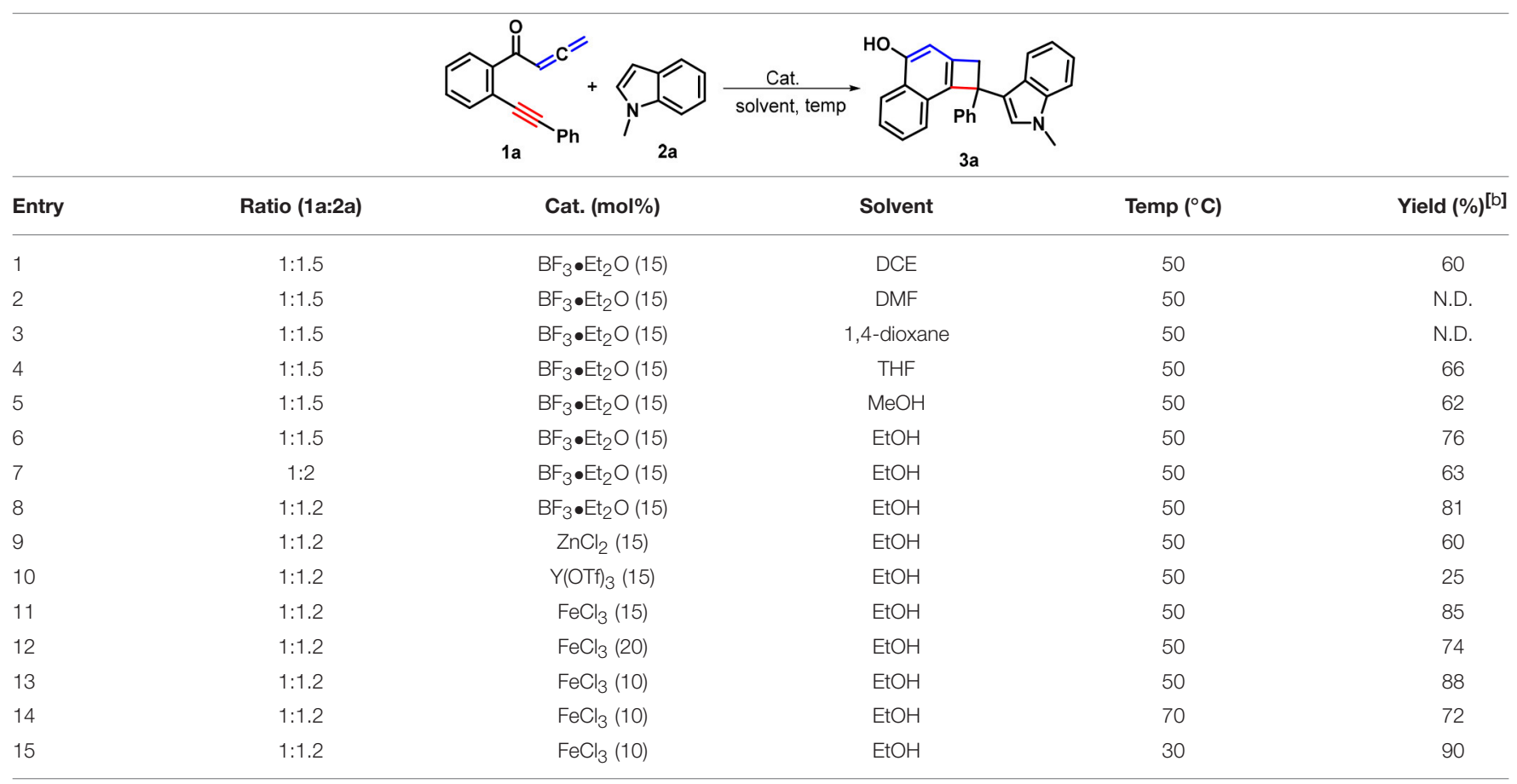

${ }^{[a]}$ Reaction conditions: Benzene-tethered yne-allenone (1a, $0.1 \mathrm{mmol}, 1.0$ equiv), $\mathrm{N}$-methylindole (2a, $x$ mmol), catalyst (y mol\%), solvent (5.0 mL), air, $8 \mathrm{~h}$

${ }^{[b]}$ /solated yield based on $\mathbf{1 a}$.

\section{MECHANISM}

Based on the above experimental observations and literature reports (Haibach et al., 2011; Li et al., 2015, 2018a; Liu et al., 2016, 2017a; Ozaki et al., 2017; Sha et al., 2018b), a feasible mechanism for forming products $\mathbf{3}$ was proposed in Scheme 3. Initially, the intramolecular [2 +2$]$ cycloaddition of yne-allenones 1 rapidly occurs to yield cyclobutene intermediate $\mathbf{A}$. In the presence of Fe-catalyst, 1,6-addition of indoles into intermediate $\mathbf{B}$ gives intermediate $\mathbf{C}$, which converts into the final products $\mathbf{3}$ through proton transfer $(\mathrm{PT})$, together with the regeneration of $\mathrm{Fe}$ catalyst.

\section{CONCLUSION}

In summary, starting from readily available yne-allenones and indoles, we have established a new Fe-catalyzed [2 + 2] cycloaddition/1,6-conjugate addition cascade for the high-efficient and benign synthesis of a variety of 3-indolyl cyclobuta $[a]$ naphthalen-4-ols with good to excellent yields. The current green protocol has the advantages of broad scope of substrates, good tolerance of functional group and high atom utilization as well as mild reaction conditions. Further application of the resulting cyclobutarenes is underway in our laboratory.

\section{MATERIALS AND METHODS}

\section{General}

All melting points are uncorrected. The NMR spectra were recorded in $\mathrm{CDCl}_{3}$ or DMSO- $d_{6}$ on a $400 \mathrm{MHz}$ instrument with
TMS as internal standard. Chemical shifts $(\delta)$ were reported in ppm with respect to TMS. Data are represented as follows: chemical shift, mutiplicity $(\mathrm{s}=$ singlet, $\mathrm{d}=$ doublet, $\mathrm{t}=$ triplet, $\mathrm{m}=$ multiplet), coupling constant $(\mathrm{J}, \mathrm{Hz})$ and integration. HRMS analyses were carried out using a TOF-MS instrument with an ESI source. X-Ray crystallographic analysis was performed with a SMART CCD and a P4 diffractometer (the copies of NMR see Supplementary Material). All yne-allenones $\mathbf{1}$ are known compounds and their preparation followed the previously reported procedures (Wei et al., 2009; Liu et al., 2017a; Li et al., 2018b).

\section{General Procedure for the Synthesis of 3 Example for the Synthesis of $3 a$}

1-(2-(Phenylethynyl)phenyl)buta-2,3-dien-1-one (1a, $0.3 \mathrm{mmol}$, $73.2 \mathrm{mg}$ ) was added to a $10-\mathrm{mL}$ reaction tubing under the air conditions. Then, $N$-methylindole (2a, $0.36 \mathrm{mmol}, 47.2 \mathrm{mg}$ ) and $\mathrm{EtOH}(5 \mathrm{~mL})$ were continuously added into the above reaction mixture. Subsequently, $\mathrm{FeCl}_{3}(10 \mathrm{~mol} \%, 4.8 \mathrm{mg}$ ) was added to the reaction system. Then the mixture was stirred at $30^{\circ} \mathrm{C}$ for $8 \mathrm{~h}$ until complete consumption of $\mathbf{1 a}$ as monitored by TLC analysis. After the reaction was finished, the reaction mixture was concentrated in vacuum, and the resulting residue was purified by column chromatography on silica gel (eluent, petroleum ether/ethyl acetate $=20: 1$ ) to afford the desired product $\mathbf{3} \mathbf{a}$ as a white solid.

\section{1-(1-methyl-1H-indol-3-yl)-1-phenyl-1,2-} dihydrocyclobuta[a]naphthalen-4-ol (3a)

White solid, $102 \mathrm{mg}$, 90\% yield; mp $179-181^{\circ} \mathrm{C} ;{ }^{1} \mathrm{H}$ NMR (400 MHz, DMSO- $\left.d_{6} ; \delta, \mathrm{ppm}\right) 10.23(\mathrm{~s}, 1 \mathrm{H}), 8.24(\mathrm{~d}, J=8.0 \mathrm{~Hz}, 1 \mathrm{H})$, 

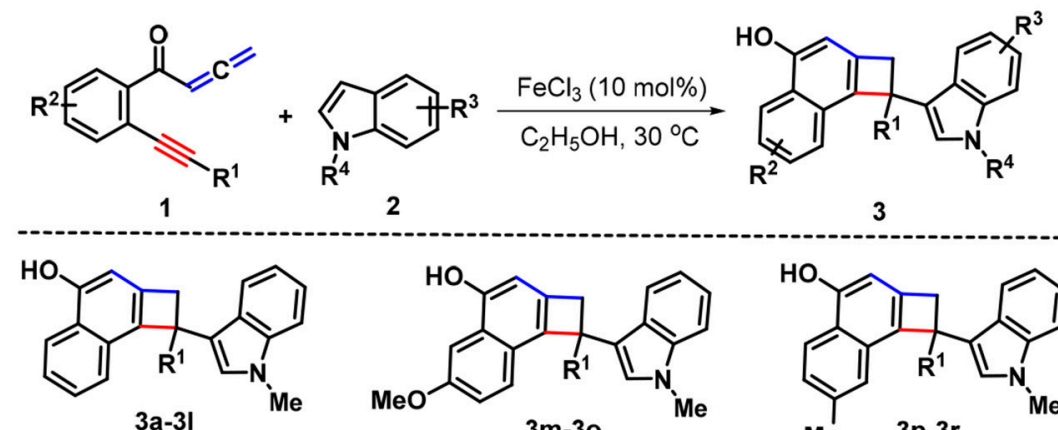

3a, $\mathrm{R}^{1}=\mathrm{Ph}(90 \%)$

3b, $\mathrm{R}^{1}=4-\mathrm{FC}_{6} \mathrm{H}_{4}(83 \%)$

3c, $\mathrm{R}^{1}=4-\mathrm{ClC}_{6} \mathrm{H}_{4}(93 \%)$

3d, $\mathrm{R}^{1}=2-\mathrm{ClC}_{6} \mathrm{H}_{4}(75 \%)$

3e, $\mathrm{R}^{1}=4-\mathrm{BrC}_{6} \mathrm{H}_{4}(98 \%)$

3f, $\mathrm{R}^{1}=p$-Tolyl $(80 \%)$

3g, $\mathbf{R}^{1}=m$-Tolyl $(85 \%)$

$3 \mathrm{~h}, \mathrm{R}^{1}=\mathrm{PMP}(86 \%)$

3i, $\mathrm{R}^{1}=4-\mathrm{EtC}_{6} \mathrm{H}_{4}(80 \%)$

3j, $\mathrm{R}^{1}=4-t-\mathrm{BuC}_{6} \mathrm{H}_{4}(76 \%)$

3k, $\mathrm{R}^{1}=1-\mathrm{Np}(77 \%)$

3
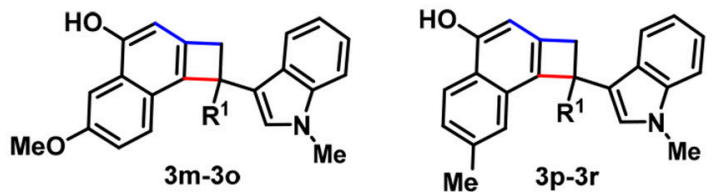

$3 \mathrm{~m}, \mathrm{R}^{1}=\mathrm{Ph}(90 \%)$

3n, $\mathrm{R}^{1}=4-\mathrm{ClC}_{6} \mathrm{H}_{4}(92 \%)$

3o, $\mathrm{R}^{1}=p$-Tolyl $(81 \%)$

$3 p, \mathrm{R}^{1}=\mathrm{Ph}(72 \%)$

3q, $\mathrm{R}^{1}=4-\mathrm{ClC}_{6} \mathrm{H}_{4}(90 \%)$

$3 \mathbf{r}, \mathrm{R}^{1}=p$-Tolyl $(79 \%)$

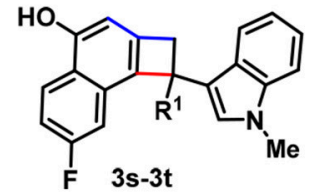

3s, $\mathrm{R}^{1}=\mathrm{Ph}(81 \%)$

3l, $\mathrm{R}^{1}=2$-Thienyl (91\%)

3t, $\mathrm{R}^{1}=4-\mathrm{ClC}_{6} \mathrm{H}_{4}(91 \%)$
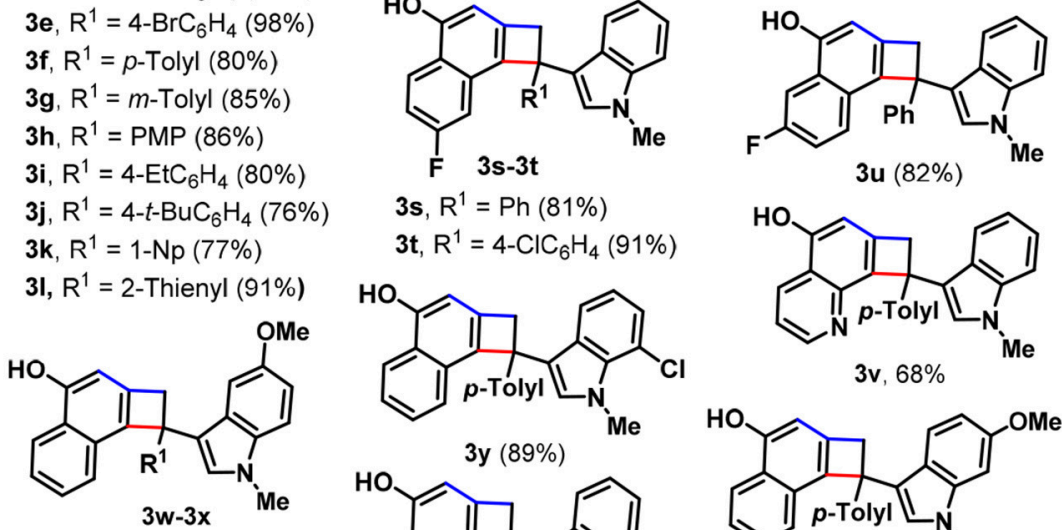

$3 w, \mathrm{R}^{1}=4-\mathrm{ClC}_{6} \mathrm{H}_{4}(92 \%)$

$3 \mathbf{x}, \mathbf{R}^{1}=p$-Tolyl $(85 \%)$

(1)

3bb (86\%)<smiles>Cc1cccc2c(C3(O)Cc4cc(O)c5ccccc5c43)cn(C)c12</smiles><smiles>COc1ccc2c(C3([14OH])Cc4cc(O)c5ccccc5c43)cn(C)c2c1</smiles>

3aa $(76 \%)$

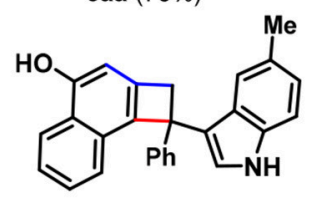

3dd $(81 \%)$

SCHEME 2 | Substrate scope for products 3.

$7.51(\mathrm{~d}, J=7.6 \mathrm{~Hz}, 1 \mathrm{H}), 7.38(\mathrm{~m}, 5 \mathrm{H}), 7.29(\mathrm{~m}, 2 \mathrm{H}), 7.21(\mathrm{~m}, 1 \mathrm{H})$, $7.07(\mathrm{~m}, 1 \mathrm{H}), 6.91-6.78(\mathrm{~m}, 4 \mathrm{H}), 3.88(\mathrm{~m}, 2 \mathrm{H}), 3.68(\mathrm{~s}, 3 \mathrm{H}) ;{ }^{13} \mathrm{C}$ NMR $\left(100 \mathrm{MHz}, \mathrm{DMSO}-d_{6} ; \delta, \mathrm{ppm}\right) 154.8,146.1,140.3,137.7$, $137.0,130.1,128.5,128.1,127.5,127.4,126.8,126.5,125.2,124.4$, $123.9,122.4,121.5,120.1,119.0,118.8,110.3,104.9,54.4,47.7$, 32.7; IR (KBr, v, cm $\left.{ }^{-1}\right) 3341,3044,1579,1474,1228,1181,1024$, 905, 806, 738; HRMS (APCI-TOF) $\mathrm{m} / \mathrm{z}$ calcd for $\mathrm{C}_{27} \mathrm{H}_{20} \mathrm{NO}$ $[\mathrm{M}-\mathrm{H}]^{-}$374.1545; found 374.1546 .

\section{1-(4-fluorophenyl)-1-(1-methyl-1H-indol-3-yl)-1,2- dihydrocyclobuta[a]naphthalen-4-ol}

(3b)

White solid, $97 \mathrm{mg}, 83 \%$ yield; $\mathrm{mp} 160-162^{\circ} \mathrm{C}$; ${ }^{1} \mathrm{H}$ NMR (400 $\left.\mathrm{MHz}, \mathrm{DMSO}-d_{6} ; \delta, \mathrm{ppm}\right) 10.24(\mathrm{~s}, 1 \mathrm{H}), 8.25(\mathrm{~d}, J=8.0 \mathrm{~Hz}$, $1 \mathrm{H}), 7.49(\mathrm{~d}, J=8.0 \mathrm{~Hz}, 1 \mathrm{H}), 7.43-7.34(\mathrm{~m}, 5 \mathrm{H}), 7.11(\mathrm{~m}, 3 \mathrm{H})$, $6.87(\mathrm{~m}, 4 \mathrm{H}), 3.88(\mathrm{~m}, 2 \mathrm{H}), 3.68(\mathrm{~s}, 3 \mathrm{H}) ;{ }^{13} \mathrm{C} \mathrm{NMR}(100 \mathrm{MHz}$, DMSO- $\left.d_{6} ; \delta, \mathrm{ppm}\right) 162.2\left({ }^{1} J_{\mathrm{CF}}=240.5 \mathrm{~Hz}\right), 159.8,154.9,142.3$ $\left({ }^{4} J_{\mathrm{CF}}=2.9 \mathrm{~Hz}\right), 142.2,140.3,137.7,136.8,129.9,129.4\left({ }^{3} J_{\mathrm{CF}}\right.$ $=7.9 \mathrm{~Hz}), 129.3,128.1,127.5,126.7,125.2,124.4,123.9,122.2$, $121.6,120.0,119.1,118.6,115.3\left({ }^{2} J_{\mathrm{CF}}=21.0 \mathrm{~Hz}\right), 115.1,110.4$, 105.0, 53.7, 47.9, 32.7; IR (KBr, $\left.v, \mathrm{~cm}^{-1}\right)$ 3399, 3051, 1573, 1464, 1226, 1188, 1014, 904, 808, 748; HRMS (APCI-TOF) m/z calcd for $\mathrm{C}_{27} \mathrm{H}_{19} \mathrm{FNO}[\mathrm{M}-\mathrm{H}]^{-}$392.1451; found 392.1453.

\section{1-(4-chlorophenyl)-1-(1-methyl-1H-indol-3-yl)-1,2- dihydrocyclobuta[a]naphthalen-4-ol \\ (3c)}

White solid, $114 \mathrm{mg}$, $93 \%$ yield; mp $170-172^{\circ} \mathrm{C} ;{ }^{1} \mathrm{H}$ NMR (400 $\mathrm{MHz}, \mathrm{DMSO}-d_{6} ; \delta$, ppm) $10.27(\mathrm{~s}, 1 \mathrm{H}), 8.24(\mathrm{~d}, J=8.0 \mathrm{~Hz}, 1 \mathrm{H})$, $7.47(\mathrm{~d}, J=7.6 \mathrm{~Hz}, 1 \mathrm{H}), 7.44-7.31(\mathrm{~m}, 7 \mathrm{H}), 7.12-7.06(\mathrm{~m}, 1 \mathrm{H})$, 


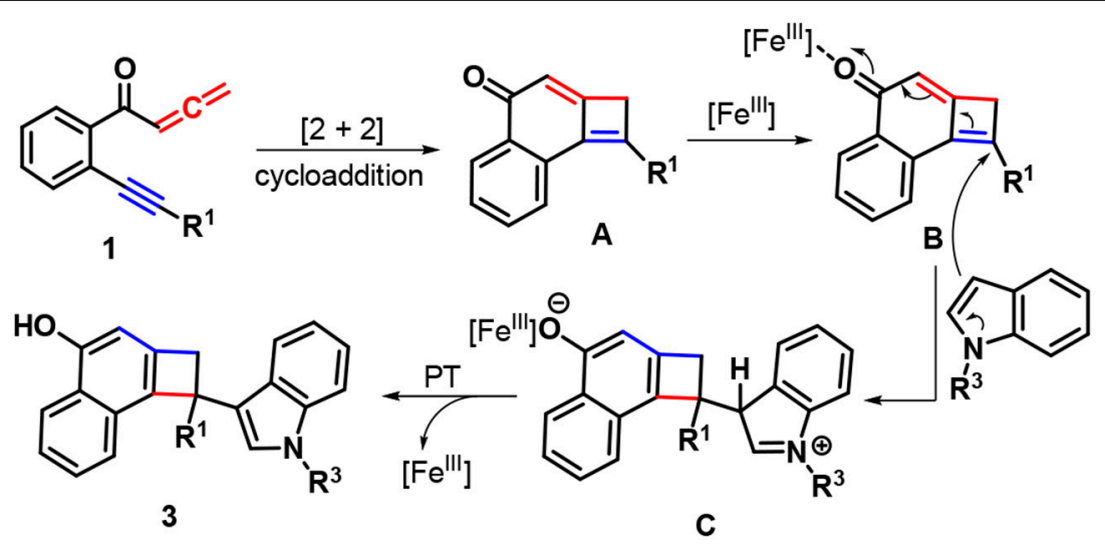

SCHEME 3 | Plausible reaction pathway.

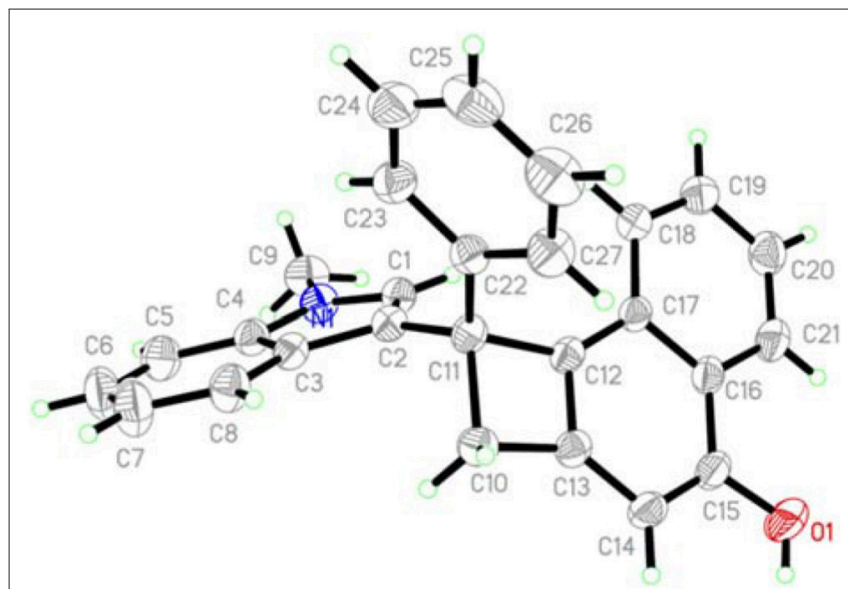

FIGURE 1 | X-Ray structure of product $\mathbf{3 a}$

$6.93(\mathrm{~s}, 1 \mathrm{H}), 6.87(\mathrm{~m}, 3 \mathrm{H}), 3.82(\mathrm{~m}, 2 \mathrm{H}), 3.69(\mathrm{~s}, 3 \mathrm{H}) ;{ }^{13} \mathrm{C} \mathrm{NMR}$ (100 MHz, DMSO- $d_{6}$; $\delta$, ppm) 154.9, 145.1, 140.3, 137.7, 136.5, $131.0,129.9,129.3,128.5,128.2,127.6,126.7,125.2,124.4,124.0$, $122.1,121.6,120.0,119.1,118.2,110.4,104.9,53.7,47.8,32.7$; IR $\left(\mathrm{KBr}, v, \mathrm{~cm}^{-1}\right) 3407,3031,1563,1441,1223,1103,1012,909,838$, 741; HRMS (APCI-TOF) m/z calcd for $\mathrm{C}_{27} \mathrm{H}_{19} \mathrm{ClNO}[\mathrm{M}-\mathrm{H}]^{-}$ 408.1156; found 408.1143.

\section{1-(2-chlorophenyl)-1-(1-methyl-1H-indol-3-yl)-1,2- dihydrocyclobuta[a]naphthalen-4-ol}

(3d)

White solid, $92 \mathrm{mg}, 75 \%$ yield; mp $177-179^{\circ} \mathrm{C}$; ${ }^{1} \mathrm{H}$ NMR (400 MHz, DMSO- $\left.d_{6} ; \delta, \mathrm{ppm}\right) 10.31(\mathrm{~s}, 1 \mathrm{H}), 8.30-8.19(\mathrm{~m}, 1 \mathrm{H}), 7.65$ $(\mathrm{d}, J=7.6 \mathrm{~Hz}, 1 \mathrm{H}), 7.53(\mathrm{~d}, J=7.6 \mathrm{~Hz}, 1 \mathrm{H}), 7.44-7.28(\mathrm{~m}, 6 \mathrm{H})$, $6.99(\mathrm{~m}, 1 \mathrm{H}), 6.93(\mathrm{~s}, 1 \mathrm{H}), 6.79(\mathrm{~s}, 1 \mathrm{H}), 6.69(\mathrm{~m}, 1 \mathrm{H}), 6.60(\mathrm{~d}$, $J=8.0 \mathrm{~Hz}, 1 \mathrm{H}), 4.32(\mathrm{~d}, J=14.4 \mathrm{~Hz}, 1 \mathrm{H}), 3.71(\mathrm{~s}, 1 \mathrm{H}), 3.66(\mathrm{~s}$, $3 \mathrm{H}) ;{ }^{13} \mathrm{C}$ NMR $\left(100 \mathrm{MHz}, \mathrm{DMSO}-d_{6} ; \delta\right.$, ppm) $155.2,143.4,140.3$, $137.7,135.9,134.0,131.0,130.5,130.1,128.9,127.9,127.6,127.4$, $126.2,125.2,124.4,123.9,122.2,121.1,119.3,118.9,116.1,110.2$, 104.7, 55.3, 47.5, 32.7; IR (KBr, $\left.v, \mathrm{~cm}^{-1}\right)$ 3442, 3022, 1533, 1421,
1203, 1123, 1010, 903, 834, 721; HRMS (APCI-TOF) m/z calcd for $\mathrm{C}_{27} \mathrm{H}_{19} \mathrm{ClNO}[\mathrm{M}-\mathrm{H}]^{-}$408.1156; found 408.1162 .

\section{1-(4-bromophenyl)-1-(1-methyl-1H-indol-3-yl)-1,2- dihydrocyclobuta[a]naphthalen-4-ol}

(3e)

White solid, $133 \mathrm{mg}, 98 \%$ yield; $\mathrm{mp} 166-168^{\circ} \mathrm{C} ;{ }^{1} \mathrm{H}$ NMR (400 $\left.\mathrm{MHz}, \mathrm{DMSO}-d_{6} ; \delta, \mathrm{ppm}\right) 10.26(\mathrm{~s}, 1 \mathrm{H}), 8.24(\mathrm{~d}, J=8.0 \mathrm{~Hz}, 1 \mathrm{H})$, $7.48(\mathrm{~d}, J=8.4 \mathrm{~Hz}, 3 \mathrm{H}), 7.43-7.34(\mathrm{~m}, 3 \mathrm{H}), 7.30(\mathrm{~d}, J=8.4 \mathrm{~Hz}$, 2H), $7.09(\mathrm{~m}, 1 \mathrm{H}), 6.93(\mathrm{~s}, 1 \mathrm{H}), 6.92-6.83(\mathrm{~m}, 3 \mathrm{H}), 3.81(\mathrm{~m}, 2 \mathrm{H})$, 3.69 (s, $3 \mathrm{H}) .{ }^{13} \mathrm{C}$ NMR (100 MHz, DMSO- $\left.d_{6} ; \delta, \mathrm{ppm}\right)$ 154.9, $145.5,140.3,137.7,136.5,131.5,129.9,129.7,128.2,127.6,126.7$, $125.3,124.4,124.0,122.1,121.6,120.0,119.6,119.1,118.1,110.4$, 104.9, 53.8, 47.8, 32.7. IR (KBr, $\left.v, \mathrm{~cm}^{-1}\right) 3412,3021,1538,1391$, 1202, 1129, 1017, 906, 818, 733; HRMS (APCI-TOF) m/z calcd for $\mathrm{C}_{27} \mathrm{H}_{19} \mathrm{BrNO}[\mathrm{M}-\mathrm{H}]^{-}$452.0651; found 452.0634 .

\section{1-(1-methyl-1H-indol-3-yl)-1-(p-tolyl)-1,2- dihydrocyclobuta[a]naphthalen-4-ol}

(3f)

White solid, $94 \mathrm{mg}, 80 \%$ yield; mp $155-157^{\circ} \mathrm{C}$; ${ }^{1} \mathrm{H}$ NMR (400 $\left.\mathrm{MHz}, \mathrm{DMSO}-d_{6} ; \delta, \mathrm{ppm}\right) 10.20(\mathrm{~s}, 1 \mathrm{H}), 8.23(\mathrm{~d}, J=8.0 \mathrm{~Hz}$, $1 \mathrm{H}), 7.49(\mathrm{~d}, J=8.0 \mathrm{~Hz}, 1 \mathrm{H}), 7.42-7.34(\mathrm{~m}, 3 \mathrm{H}), 7.26(\mathrm{~d}, J=$ $8.0 \mathrm{~Hz}, 2 \mathrm{H}), 7.08(\mathrm{~d}, J=8.0 \mathrm{~Hz}, 3 \mathrm{H}), 6.92-6.79(\mathrm{~m}, 4 \mathrm{H}), 3.80$ $(\mathrm{m}, 2 \mathrm{H}), 3.68(\mathrm{~s}, 3 \mathrm{H}), 2.26(\mathrm{~s}, 3 \mathrm{H}) ;{ }^{13} \mathrm{C}$ NMR (100 MHz, DMSO$\left.d_{6} ; \delta, \mathrm{ppm}\right) 154.7,143.0,140.3,137.7,137.2,135.3,130.0,129.1$, 128.0, 127.4, 127.3, 126.8, 125.2, 124.3, 123.8, 122.4, 121.5, 120.2, $119.0,118.9,110.3,105.0,54.0,47.8,32.7,21.1$; IR $\left(\mathrm{KBr}, v, \mathrm{~cm}^{-1}\right)$ $3418,3009,1541,1401,1192,1121,1012,916,813,730$; HRMS (APCI-TOF) $\mathrm{m} / \mathrm{z}$ calcd for $\mathrm{C}_{28} \mathrm{H}_{22} \mathrm{NO}[\mathrm{M}-\mathrm{H}]^{-} 388.1702$; found 388.1723.

\section{1-(1-methyl-1H-indol-3-yl)-1-(m-tolyl)-1,2-} dihydrocyclobuta[a]naphthalen-4-ol

(3g)

White solid, $99 \mathrm{mg}, 85 \%$ yield; $\mathrm{mp} 149-151{ }^{\circ} \mathrm{C} ;{ }^{1} \mathrm{H}$ NMR (400 $\left.\mathrm{MHz}, \mathrm{DMSO}-d_{6} ; \delta, \mathrm{ppm}\right) 10.20(\mathrm{~s}, 1 \mathrm{H}), 8.23(\mathrm{~d}, J=8.4 \mathrm{~Hz}, 1 \mathrm{H})$, $7.51(\mathrm{~d}, J=8.0 \mathrm{~Hz}, 1 \mathrm{H}), 7.38(\mathrm{~m}, 3 \mathrm{H}), 7.23(\mathrm{~s}, 1 \mathrm{H}), 7.18(\mathrm{~d}, J$ $=5.6 \mathrm{~Hz}, 2 \mathrm{H}), 7.09-7.01(\mathrm{~m}, 2 \mathrm{H}), 6.85(\mathrm{~m}, 4 \mathrm{H}), 3.82(\mathrm{~m}, 2 \mathrm{H})$, 
3.68 (s, 3H), 2.22 (s, 3H); ${ }^{13} \mathrm{C}$ NMR (100 MHz, DMSO- $d_{6} ; \delta$, ppm) 154.7, 146.0, 140.3, 137.7, 137.4, 137.1, 128.4, 128.0, 127.4, $127.2,126.8,125.2,124.8,124.3,123.8,120.1,118.9,110.3,105.0$, 54.3, 47.7, 32.7, 21.8; IR (KBr, v, $\left.\mathrm{cm}^{-1}\right)$ 3411, 2966, 1511, 1406, 1162, 1091, 1015, 911, 833, 727; HRMS (APCI-TOF) m/z calcd for $\mathrm{C}_{28} \mathrm{H}_{22} \mathrm{NO}[\mathrm{M}-\mathrm{H}]^{-}$388.1702; found 388.1720.

\section{1-(4-methoxyphenyl)-1-(1-methyl-1H-indol-3-yl)-1,2- dihydrocyclobuta[a]naphthalen-4-ol \\ (3h)}

White solid, $105 \mathrm{mg}$, $86 \%$ yield; mp $167-169^{\circ} \mathrm{C} ;{ }^{1} \mathrm{H}$ NMR (400 $\left.\mathrm{MHz}, \mathrm{DMSO}-d_{6} ; \delta, \mathrm{ppm}\right) 10.19(\mathrm{~s}, 1 \mathrm{H}), 8.25(\mathrm{~d}, J=8.0 \mathrm{~Hz}, 1 \mathrm{H})$, $7.50(\mathrm{~d}, J=8.0 \mathrm{~Hz}, 1 \mathrm{H}), 7.42-7.33(\mathrm{~m}, 3 \mathrm{H}), 7.29(\mathrm{~d}, J=8.8 \mathrm{~Hz}$, $2 \mathrm{H}), 7.07(\mathrm{~m}, 1 \mathrm{H}), 6.91-6.81(\mathrm{~m}, 6 \mathrm{H}), 3.79(\mathrm{~m}, 2 \mathrm{H}), 3.70(\mathrm{~s}, 3 \mathrm{H})$, $3.67(\mathrm{~s}, 3 \mathrm{H}) ;{ }^{13} \mathrm{C}$ NMR $\left(100 \mathrm{MHz}\right.$, DMSO- $d_{6} ; \delta$, ppm) 157.9, $154.7,140.3,138.1,137.7,137.3,130.0,128.5,128.0,127.3,126.8$, 125.2, 124.4, 123.8, 122.4, 121.5, 120.2, 119.2, 118.9, 113.8, 110.3, 105.0, 55.4, 53.7, 47.9, 32.7; IR (KBr, $\left.v, \mathrm{~cm}^{-1}\right)$ 3417, 2996, 1517, 1403, 1177, 1096, 1013, 914, 845, 720; HRMS (APCI-TOF) m/z calcd for $\mathrm{C}_{28} \mathrm{H}_{22} \mathrm{NO}_{2}[\mathrm{M}-\mathrm{H}]^{-}$404.1651; found 404.1638 .

\section{1-(4-ethylphenyl)-1-(1-methyl-1H-indol-3-yl)-1,2- dihydrocyclobuta[a]naphthalen-4-ol}

(3i)

White solid, $97 \mathrm{mg}, 80 \%$ yield; mp $165-167^{\circ} \mathrm{C} ;{ }^{1} \mathrm{H}$ NMR (400 $\left.\mathrm{MHz}, \mathrm{DMSO}-d_{6} ; \delta, \mathrm{ppm}\right) 10.19(\mathrm{~s}, 1 \mathrm{H}), 8.24(\mathrm{~d}, J=8.0 \mathrm{~Hz}, 1 \mathrm{H})$, $7.50(\mathrm{~d}, J=8.0 \mathrm{~Hz}, 1 \mathrm{H}), 7.42-7.33(\mathrm{~m}, 3 \mathrm{H}), 7.29(\mathrm{~d}, J=8.0 \mathrm{~Hz}$, $2 \mathrm{H}), 7.13-7.04(\mathrm{~m}, 3 \mathrm{H}), 6.90-6.77(\mathrm{~m}, 4 \mathrm{H}), 3.80(\mathrm{~m}, 2 \mathrm{H}), 3.68(\mathrm{~s}$, $3 \mathrm{H}), 2.56(\mathrm{~m}, 2 \mathrm{H}), 1.15(\mathrm{~m}, 3 \mathrm{H}) ;{ }^{13} \mathrm{C}$ NMR $(100 \mathrm{MHz}$, DMSO$\left.d_{6} ; \delta, \mathrm{ppm}\right) 154.7,143.3,141.7,140.3,137.7,137.2,130.0,128.0$, $127.9,127.4,127.4,126.8,125.2,124.3,123.8,122.4,121.5,120.2$, $119.0,118.9,110.3,105.0,54.0,47.8,32.7,28.2,16.0$; IR (KBr, $v$, $\mathrm{cm}^{-1}$ ) 3387, 3013, 1510, 1406, 1167, 1091, 1012, 918, 832, 722; HRMS (APCI-TOF) $\mathrm{m} / \mathrm{z}$ calcd for $\mathrm{C}_{29} \mathrm{H}_{24} \mathrm{NO}[\mathrm{M}-\mathrm{H}]^{-} 402.1858$; found 402.1874 .

\section{1-(4-(tert-butyl)phenyl)-1-(1-methyl-1H-indol-3-yl)- 1,2-dihydrocyclobuta[a]naphthalen-4-ol}

(3j)

White solid, $104 \mathrm{mg}, 80 \%$ yield; mp $178-180^{\circ} \mathrm{C} ;{ }^{1} \mathrm{H}$ NMR (400 $\left.\mathrm{MHz}, \mathrm{DMSO}-d_{6} ; \delta, \mathrm{ppm}\right) 10.19(\mathrm{~s}, 1 \mathrm{H}), 8.24(\mathrm{~d}, J=8.4 \mathrm{~Hz}, 1 \mathrm{H})$, $7.53(\mathrm{~d}, J=8.0 \mathrm{~Hz}, 1 \mathrm{H}), 7.35(\mathrm{~m}, 7 \mathrm{H}), 7.07(\mathrm{~m}, 1 \mathrm{H}), 6.93-6.78$ (m, 4H), $3.81(\mathrm{~m}, 2 \mathrm{H}), 3.68(\mathrm{~s}, 3 \mathrm{H}), 1.25(\mathrm{~s}, 9 \mathrm{H}) ;{ }^{13} \mathrm{C}$ NMR $(100$ MHz, DMSO- $d_{6}$; $\delta$, ppm) 154.7, 148.5, 143.0, 140.3, 137.7, 137.1, $130.1,128.0,127.4,127.2,126.8,125.3,125.2,124.3,123.8,122.5$, $121.5,120.2,119.0,118.9,110.3,104.9,54.0,47.7,34.5,32.7,31.6$; IR $\left(\mathrm{KBr}, v, \mathrm{~cm}^{-1}\right) 3402,3010,1512,1423,1177,1093,1018,933$, 814, 711; HRMS (APCI-TOF) $\mathrm{m} / \mathrm{z}$ calcd for $\mathrm{C}_{31} \mathrm{H}_{28} \mathrm{NO}[\mathrm{M}-\mathrm{H}]^{-}$ 430.2171; found 430.2187 .

\section{1-(1-methyl-1H-indol-3-yl)-1-(naphthalen-1-yl)-1,2- dihydrocyclobuta[a]naphthalen-4-ol \\ (3k)}

White solid, $98 \mathrm{mg}$, $77 \%$ yield; mp $162-164^{\circ} \mathrm{C} ;{ }^{1} \mathrm{H}$ NMR (400 MHz, DMSO- $\left.d_{6} ; \delta, \mathrm{ppm}\right) 10.25(\mathrm{~s}, 1 \mathrm{H}), 8.27(\mathrm{~d}, J=8.0 \mathrm{~Hz}, 1 \mathrm{H})$, 7.89-7.81 (m, 3H), $7.71(\mathrm{~d}, J=7.2 \mathrm{~Hz}, 1 \mathrm{H}), 7.57(\mathrm{~m}, 2 \mathrm{H}), 7.47-$ $7.34(\mathrm{~m}, 5 \mathrm{H}), 7.08(\mathrm{~m}, 1 \mathrm{H}), 6.95(\mathrm{~s}, 1 \mathrm{H}), 6.91(\mathrm{~d}, J=6.0 \mathrm{~Hz}$,
2H), $6.81(\mathrm{~m}, 1 \mathrm{H}), 3.93(\mathrm{~s}, 2 \mathrm{H}), 3.69(\mathrm{~m}, 3 \mathrm{H}) ;{ }^{13} \mathrm{C}$ NMR $(100$ MHz, DMSO- $d_{6} ; \delta$, ppm) 154.9, 143.7, 140.4, 137.8, 137.1, 133.3, $132.1,130.1,128.2,128.1,127.8,127.5,126.9,126.6,126.5,126.0$, $125.3,125.2,124.4,123.9,122.3,121.5,120.1,119.0,118.6,110.4$, 105.1, 54.5, 47.5, 32.7; IR (KBr, $\left.v, \mathrm{~cm}^{-1}\right) 3422,3014,1510,1413$, 1171, 1088, 1015, 937, 825, 727; HRMS (APCI-TOF) m/z calcd for $\mathrm{C}_{31} \mathrm{H}_{22} \mathrm{NO}[\mathrm{M}-\mathrm{H}]^{-}$424.1702; found 424.1713 .

\section{1-(1-methyl-1H-indol-3-yl)-1-(thiophen-2-yl)-1,2- dihydrocyclobuta[a]naphthalen-4-ol \\ (3I)}

White solid, $104 \mathrm{mg}, 91 \%$ yield; mp $158-160{ }^{\circ} \mathrm{C} ;{ }^{1} \mathrm{H}$ NMR (400 $\left.\mathrm{MHz}, \mathrm{DMSO}-d_{6} ; \delta, \mathrm{ppm}\right) 10.26(\mathrm{~s}, 1 \mathrm{H}), 8.26(\mathrm{~d}, J=8.4 \mathrm{~Hz}, 1 \mathrm{H})$, $7.71(\mathrm{~d}, J=8.4 \mathrm{~Hz}, 1 \mathrm{H}), 7.48(\mathrm{~m}, 1 \mathrm{H}), 7.43-7.36(\mathrm{~m}, 2 \mathrm{H}), 7.31$ $(\mathrm{d}, J=4.8 \mathrm{~Hz}, 1 \mathrm{H}), 7.12(\mathrm{~m}, 2 \mathrm{H}), 6.99(\mathrm{~d}, J=5.6 \mathrm{~Hz}, 2 \mathrm{H}), 6.92$ $(\mathrm{m}, 2 \mathrm{H}), 6.85(\mathrm{~s}, 1 \mathrm{H}), 3.98(\mathrm{~d}, J=13.6 \mathrm{~Hz}, 1 \mathrm{H}), 3.77(\mathrm{~d}, J=$ $13.6 \mathrm{~Hz}, 1 \mathrm{H}), 3.69(\mathrm{~s}, 3 \mathrm{H}) ;{ }^{13} \mathrm{C}$ NMR $\left(100 \mathrm{MHz}\right.$, DMSO- $d_{6}$; $\delta$, ppm) 155.0, 151.2, 140.1, 137.6, 136.8, 129.5, 127.5, 126.9, 126.6, 125.3, 124.6, 124.5, 124.4, 124.0, 122.4, 121.6, 120.2, 119.1, 119.0, 110.4, 105.0, 50.9, 48.9, 32.7; IR $\left(\mathrm{KBr}, v, \mathrm{~cm}^{-1}\right) 3427,3050,1517$, 1421, 1178, 1068, 1020, 936, 821, 734; HRMS (APCI-TOF) m/z calcd for $\mathrm{C}_{25} \mathrm{H}_{18} \mathrm{NOS}[\mathrm{M}-\mathrm{H}]^{-} 380.1110$; found 380.1108 .

\section{6-methoxy-1-(1-methyl-1H-indol-3-yl)-1-phenyl-1,2- dihydrocyclobuta[a]naphthalen-4-ol \\ (3m)}

White solid, $110 \mathrm{mg}$, $90 \%$ yield; mp $172-174^{\circ} \mathrm{C} ;{ }^{1} \mathrm{H}$ NMR (400 $\left.\mathrm{MHz}, \mathrm{DMSO}-d_{6} ; \delta, \mathrm{ppm}\right) 10.11(\mathrm{~s}, 1 \mathrm{H}), 7.58(\mathrm{~d}, J=2.4 \mathrm{~Hz}, 1 \mathrm{H})$, $7.43(\mathrm{~d}, J=8.8 \mathrm{~Hz}, 1 \mathrm{H}), 7.37(\mathrm{~m}, 3 \mathrm{H}), 7.29(\mathrm{~m}, 2 \mathrm{H}), 7.20(\mathrm{~m}, 1 \mathrm{H})$, 7.11-7.05 (m, 2H), 6.88-6.79 (m, $4 \mathrm{H}), 3.87(\mathrm{~d}, J=13.2 \mathrm{~Hz}, 1 \mathrm{H})$, $3.84(\mathrm{~s}, 3 \mathrm{H}), 3.76(\mathrm{~d}, J=13.6 \mathrm{~Hz}, 1 \mathrm{H}), 3.68(\mathrm{~s}, 3 \mathrm{H}) ;{ }^{13} \mathrm{C} \mathrm{NMR}$ (100 MHz, DMSO- $\left.d_{6} ; \delta, \mathrm{ppm}\right) 156.1,153.7,146.1,137.7,137.3$, $137.2,128.5,128.1,127.5,126.8,126.4,126.2,125.5,124.0,121.5$, 120.1, 119.6, 118.9, 110.3, 105.4, 103.2, 55.5, 54.3, 47.7, 32.7; IR $\left(\mathrm{KBr}, v, \mathrm{~cm}^{-1}\right) 3387,3045,1507,1422,1172,1058,1011,932$, 827, 739; HRMS (APCI-TOF) $\mathrm{m} / \mathrm{z}$ calcd for $\mathrm{C}_{28} \mathrm{H}_{22} \mathrm{NO}_{2}[\mathrm{M}-\mathrm{H}]^{-}$ 404.1651; found 404.1630.

\section{1-(4-chlorophenyl)-6-methoxy-1-(1-methyl-1H-indol-} 3-yl)-1,2-dihydrocyclobuta[a]naphthalen-4-ol

(3n)

White solid, $121 \mathrm{mg}, 92 \%$ yield; mp $175-177^{\circ} \mathrm{C} ;{ }^{1} \mathrm{H}$ NMR (400 $\left.\mathrm{MHz}, \mathrm{DMSO}-d_{6} ; \delta, \mathrm{ppm}\right) 10.15(\mathrm{~s}, 1 \mathrm{H}), 7.59(\mathrm{~d}, J=2.4 \mathrm{~Hz}, 1 \mathrm{H})$, 7.44-7.31 (m, 6H), 7.13-7.05 (m, 2H), 6.94-6.81 (m, 4H), 3.85 (s, 3H), 3.83-3.72 (m, 2H), 3.68 (s, 3H); ${ }^{13} \mathrm{C}$ NMR (100 MHz, DMSO- $d_{6} ; \delta$, ppm) $156.2,153.9,145.1,137.7,137.2,136.7,131.0$, $129.3,128.5,128.1,126.7,126.3,125.3,123.8,121.6,120.0,119.7$, 119.1, 118.3, 110.4, 105.4, 103.2, 55.5, 53.7, 47.7, 32.7. IR ( $\mathrm{KBr}$, $\left.v, \mathrm{~cm}^{-1}\right) 3382,3025,1523,1421,1192,1074,1008,934,828$, 736. HRMS (APCI-TOF) $\mathrm{m} / \mathrm{z}$ calcd for $\mathrm{C}_{28} \mathrm{H}_{21} \mathrm{NClO}_{2}[\mathrm{M}-\mathrm{H}]^{-}$ 438.1261; found 438.1277 .

\section{6-methoxy-1-(1-methyl-1H-indol-3-yl)-1-(p-tolyl)-1,2- dihydrocyclobuta[a]naphthalen-4-ol}

(3o)

White solid, $102 \mathrm{mg}, 81 \%$ yield; mp $170-172^{\circ} \mathrm{C} ;{ }^{1} \mathrm{H}$ NMR (400 $\left.\mathrm{MHz}, \mathrm{DMSO}-d_{6} ; \delta, \mathrm{ppm}\right) 10.09$ (s, $\left.1 \mathrm{H}\right), 7.59(\mathrm{~d}, J=2.4 \mathrm{~Hz}, 1 \mathrm{H})$, 
$7.43(\mathrm{~d}, J=9.2 \mathrm{~Hz}, 1 \mathrm{H}), 7.35(\mathrm{~d}, J=8.0 \mathrm{~Hz}, 1 \mathrm{H}), 7.26(\mathrm{~d}, J=$ $8.0 \mathrm{~Hz}, 2 \mathrm{H}), 7.07$ (m, 4H), 6.89-6.80 (m, 4H), 3.84 (s, 3H), 3.83$3.72(\mathrm{~m}, 2 \mathrm{H}), 3.67(\mathrm{~s}, 3 \mathrm{H}), 2.26(\mathrm{~s}, 3 \mathrm{H}) ;{ }^{13} \mathrm{C} \mathrm{NMR}(100 \mathrm{MHz}$, DMSO- $d_{6} ; \delta$, ppm) $156.1,153.6,143.1,137.7,137.4,137.3,135.3$, $129.1,128.0,127.4,126.8,126.2,125.5,124.0,121.5,120.2,119.5$, $119.1,118.9,110.2,105.4,103.1,55.5,54.0,47.7,32.6,21.1$; IR $\left(\mathrm{KBr}, v, \mathrm{~cm}^{-1}\right) 3387,3022,1521,1425,1202,1094,1016,942$, 835, 721; HRMS (APCI-TOF) $\mathrm{m} / \mathrm{z}$ calcd for $\mathrm{C}_{29} \mathrm{H}_{24} \mathrm{NO}_{2}[\mathrm{M}-\mathrm{H}]^{-}$ 418.1808; found 418.1822.

\section{7-methyl-1-(1-methyl-1H-indol-3-yl)-1-phenyl-1,2- dihydrocyclobuta[a]naphthalen-4-ol \\ (3p)}

White solid, $84 \mathrm{mg}, 72 \%$ yield; mp $147-149^{\circ} \mathrm{C} ;{ }^{1} \mathrm{H}$ NMR (400 MHz, DMSO- $\left.d_{6} ; \delta, \mathrm{ppm}\right) 10.12(\mathrm{~s}, 1 \mathrm{H}), 8.14(\mathrm{~d}, J=8.8 \mathrm{~Hz}, 1 \mathrm{H})$, $7.38(\mathrm{~m}, 3 \mathrm{H}), 7.32-7.26(\mathrm{~m}, 3 \mathrm{H}), 7.20(\mathrm{~m}, 2 \mathrm{H}), 7.08(\mathrm{~m}, 1 \mathrm{H}), 6.88$ $(\mathrm{d}, J=4.4 \mathrm{~Hz}, 2 \mathrm{H}), 6.86-6.77(\mathrm{~m}, 2 \mathrm{H}), 3.86(\mathrm{~d}, J=13.6 \mathrm{~Hz}, 1 \mathrm{H})$, $3.73(\mathrm{~d}, J=13.6 \mathrm{~Hz}, 1 \mathrm{H}), 3.69(\mathrm{~s}, 3 \mathrm{H}), 2.32(\mathrm{~s}, 3 \mathrm{H}) ;{ }^{13} \mathrm{C}$ NMR $(100$ $\left.\mathrm{MHz}, \mathrm{DMSO}-d_{6} ; \delta, \mathrm{ppm}\right) 154.8,146.2,140.5,137.7,136.6,136.3$, $130.4,128.5,128.1,127.5,126.8,126.4,126.0,124.3,123.5,121.5$, $121.3,120.2,118.9,118.9,110.3,104.2,54.4,47.9,32.7,22.0$; IR $\left(\mathrm{KBr}, v, \mathrm{~cm}^{-1}\right) 3507,3052,1522,1420,1201,1098,1015,944$, 841, 727; HRMS (APCI-TOF) m/z calcd for $\mathrm{C}_{28} \mathrm{H}_{22} \mathrm{NO}[\mathrm{M}-\mathrm{H}]^{-}$ 388.1702; found 388.1727.

\section{1-(4-chlorophenyl)-7-methyl-1-(1-methyl-1H-indol-3- yl)-1,2-dihydrocyclobuta[a]naphthalen-4-ol}

(3q)

White solid, $114 \mathrm{mg}, 90 \%$ yield; mp $152-154^{\circ} \mathrm{C} ;{ }^{1} \mathrm{H}$ NMR (400 $\left.\mathrm{MHz}, \mathrm{DMSO}-d_{6} ; \delta, \mathrm{ppm}\right) 10.16(\mathrm{~s}, 1 \mathrm{H}), 8.14(\mathrm{~d}, J=8.4 \mathrm{~Hz}, 1 \mathrm{H})$, 7.40-7.33 (m, 5H), 7.25 (s, 1H), 7.19 (d, $J=8.8 \mathrm{~Hz}, 1 \mathrm{H}), 7.09$ $(\mathrm{m}, 1 \mathrm{H}), 6.94-6.83(\mathrm{~m}, 3 \mathrm{H}), 6.79(\mathrm{~s}, 1 \mathrm{H}), 3.85(\mathrm{~d}, J=13.6 \mathrm{~Hz}$, $1 \mathrm{H}), 3.73(\mathrm{~s}, 1 \mathrm{H}), 3.70(\mathrm{~s}, 3 \mathrm{H}), 2.33(\mathrm{~s}, 3 \mathrm{H}) ;{ }^{13} \mathrm{C} \mathrm{NMR}(100 \mathrm{MHz}$, DMSO- $d_{6} ; \delta$, ppm) 154.9, 145.2, 140.4, 137.7, 136.7, 135.9, 131.0, $130.2,129.4,128.5,128.2,126.7,126.1,124.4,123.5,121.6,121.1$, $120.1,119.1,118.3,110.4,104.2,53.7,47.9,32.7,22.0$; IR (KBr, $\left.v, \mathrm{~cm}^{-1}\right) 3495,3042,1520,1402,1221,1084,1016,947,831$, 709; HRMS (APCI-TOF) $\mathrm{m} / \mathrm{z}$ calcd for $\mathrm{C}_{28} \mathrm{H}_{21} \mathrm{ClNO}[\mathrm{M}-\mathrm{H}]^{-}$ 422.1312; found 422.1314 .

\section{7-methyl-1-(1-methyl-1H-indol-3-yl)-1-(p-tolyl)-1,2- dihydrocyclobuta[a]naphthalen-4-ol \\ (3r)}

White solid, $96 \mathrm{mg}, 79 \%$ yield; mp $153-155^{\circ} \mathrm{C}$; ${ }^{1} \mathrm{H}$ NMR (400 $\left.\mathrm{MHz}, \mathrm{DMSO}-d_{6} ; \delta, \mathrm{ppm}\right) 10.10(\mathrm{~s}, 1 \mathrm{H}), 8.14(\mathrm{~d}, J=8.4 \mathrm{~Hz}, 1 \mathrm{H})$, $7.36(\mathrm{~d}, J=8.4 \mathrm{~Hz}, 1 \mathrm{H}), 7.26(\mathrm{~d}, J=8.0 \mathrm{~Hz}, 3 \mathrm{H}), 7.18(\mathrm{~d}, J=$ $8.8 \mathrm{~Hz}, 1 \mathrm{H}), 7.08(\mathrm{~m}, 3 \mathrm{H}), 6.90(\mathrm{~d}, J=8.8 \mathrm{~Hz}, 2 \mathrm{H}), 6.83(\mathrm{~m}, 1 \mathrm{H})$, $6.79(\mathrm{~s}, 1 \mathrm{H}), 3.88-3.69(\mathrm{~m}, 2 \mathrm{H}), 3.68(\mathrm{~s}, 3 \mathrm{H}), 2.32(\mathrm{~s}, 3 \mathrm{H}), 2.26(\mathrm{~s}$, $3 \mathrm{H}) ;{ }^{13} \mathrm{C}$ NMR $\left(100 \mathrm{MHz}, \mathrm{DMSO}-d_{6}\right.$; $\delta$, ppm) 154.7, 143.1, 140.4, $137.7,136.5,136.5,135.3,130.3,129.1,128.0,127.4,126.9,126.0$, $124.3,123.4,121.5,121.3,120.3,119.0,118.9,110.3,104.2,54.0$, 47.9, 32.7, 22.0, 21.1; IR (KBr, v, cm $\left.{ }^{-1}\right)$ 3490, 3031, 1500, 1422, 1213, 1090, 1012, 948, 843, 719; HRMS (APCI-TOF) m/z calcd for $\mathrm{C}_{29} \mathrm{H}_{24} \mathrm{NO}[\mathrm{M}-\mathrm{H}]^{-}$402.1858; found 402.1844.

\section{7-fluoro-1-(1-methyl-1H-indol-3-yl)-1-phenyl-1,2- dihydrocyclobuta[a]naphthalen-4-ol \\ (3s)}

White solid, $96 \mathrm{mg}, 81 \%$ yield; $\mathrm{mp} 144-146^{\circ} \mathrm{C} ;{ }^{1} \mathrm{H}$ NMR (400 $\left.\mathrm{MHz}, \mathrm{DMSO}-d_{6} ; \delta, \mathrm{ppm}\right) 10.36(\mathrm{~s}, 1 \mathrm{H}), 7.87(\mathrm{~m}, 1 \mathrm{H}), 7.55(\mathrm{~m}$, $1 \mathrm{H}), 7.33(\mathrm{~m}, 6 \mathrm{H}), 7.21(\mathrm{~m}, 1 \mathrm{H}), 7.08(\mathrm{~m}, 1 \mathrm{H}), 6.93(\mathrm{~s}, 1 \mathrm{H})$, 6.91-6.77 (m, 3H), $3.84(\mathrm{~m}, 2 \mathrm{H}), 3.68(\mathrm{~s}, 3 \mathrm{H}) .{ }^{13} \mathrm{C}$ NMR (100 $\left.\mathrm{MHz}, \mathrm{DMSO}-d_{6} ; \delta, \mathrm{ppm}\right) 160.3\left({ }^{1} J_{\mathrm{CF}}=239.4 \mathrm{~Hz}\right), 157.9,154.2$, $154.1,145.8,139.7\left({ }^{6} J_{\mathrm{CF}}=2.4 \mathrm{~Hz}\right), 139.6,137.7,137.3,128.6$, $128.1,127.5,127.2,126.8,126.7\left({ }^{3} J_{\mathrm{CF}}=19.7 \mathrm{~Hz}\right), 125.9\left({ }^{5} J_{\mathrm{CF}}=\right.$ $8.1 \mathrm{~Hz}), 125.8,125.1\left({ }^{4} J_{\mathrm{CF}}=8.4 \mathrm{~Hz}\right), 125.0,121.5,120.0,119.0$, $118.6,117.5,117.3,110.3,108.0\left({ }^{2} J_{\mathrm{CF}}=21.7 \mathrm{~Hz}\right), 107.8,106.0$, 54.4, 47.8, 32.7; IR ( $\left.\mathrm{KBr}, v, \mathrm{~cm}^{-1}\right)$ 3501, 3021, 1502, 1421, 1215, 1099, 1014, 962, 883, 712; HRMS (APCI-TOF) $\mathrm{m} / \mathrm{z}$ calcd for $\mathrm{C}_{27} \mathrm{H}_{19} \mathrm{FNO}[\mathrm{M}-\mathrm{H}]^{-}$392.1451; found 392.1459.

\section{1-(4-chlorophenyl)-7-fluoro-1-(1-methyl-1H-indol-3- yl)-1,2-dihydrocyclobuta[a]naphthalen-4-ol}

(3t)

White solid, $117 \mathrm{mg}, 91 \%$ yield; $\mathrm{mp} 149-151^{\circ} \mathrm{C} ;{ }^{1} \mathrm{H}$ NMR (400 MHz, DMSO- $\left.d_{6} ; \delta, \mathrm{ppm}\right) 10.44(\mathrm{~s}, 1 \mathrm{H}), 8.30(\mathrm{~m}, 1 \mathrm{H}), 7.41-7.32$ (m, 5H), $7.24(\mathrm{~m}, 1 \mathrm{H}), 7.12-7.04(\mathrm{~m}, 2 \mathrm{H}), 6.98(\mathrm{~s}, 1 \mathrm{H}), 6.86(\mathrm{~d}, J$ $=13.2 \mathrm{~Hz}, 3 \mathrm{H}), 3.82(\mathrm{~m}, 2 \mathrm{H}), 3.71(\mathrm{~s}, 3 \mathrm{H}) ;{ }^{13} \mathrm{C}$ NMR $(100 \mathrm{MHz}$, DMSO- $d_{6} ; \delta$, ppm) $160.0\left({ }^{1} J_{\mathrm{CF}}=231.6 \mathrm{~Hz}\right), 157.1,155.3,144.8$, $142.4,137.8,136.2\left({ }^{6} J_{\mathrm{CF}}=3.4 \mathrm{~Hz}\right), 136.1,131.1,130.5\left({ }^{5} J_{\mathrm{CF}}=\right.$ $9.6 \mathrm{~Hz}), 130.4,129.3,128.6,128.2,127.8\left({ }^{4} J_{\mathrm{CF}}=9.9 \mathrm{~Hz}\right), 126.6$, $122.4,121.6,119.9,119.2,117.8,113.9\left({ }^{2} J_{\mathrm{CF}}=24.1 \mathrm{~Hz}\right), 113.6$, $110.5,105.5\left({ }^{3} J_{\mathrm{CF}}=20.3 \mathrm{~Hz}\right), 105.3,104.5,53.7,47.7,32.7$; IR $\left(\mathrm{KBr}, v, \mathrm{~cm}^{-1}\right) 3512,3020,1505,1411,1210,1095,1010,966,861$, 740; HRMS (APCI-TOF) $\mathrm{m} / \mathrm{z}$ calcd for $\mathrm{C}_{27} \mathrm{H}_{18} \mathrm{ClFNO}[\mathrm{M}-\mathrm{H}]^{-}$ 426.1061; found 426.1066 .

\section{6-fluoro-1-(1-methyl-1H-indol-3-yl)-1-phenyl-1,2- dihydrocyclobuta[a]naphthalen-4-ol}

(3u)

White solid, $97 \mathrm{mg}, 82 \%$ yield; $\mathrm{mp} 148-150{ }^{\circ} \mathrm{C} ;{ }^{1} \mathrm{H}$ NMR (400 $\left.\mathrm{MHz}, \mathrm{DMSO}-d_{6} ; \delta, \mathrm{ppm}\right) 10.41(\mathrm{~s}, 1 \mathrm{H}), 8.30(\mathrm{~m}, 1 \mathrm{H}), 7.38(\mathrm{~d}$, $J=8.8 \mathrm{~Hz}, 3 \mathrm{H}), 7.30(\mathrm{~m}, 2 \mathrm{H}), 7.24(\mathrm{~m}, 2 \mathrm{H}), 7.08(\mathrm{~m}, 2 \mathrm{H}), 6.94(\mathrm{~s}$, $1 \mathrm{H}), 6.90-6.79(\mathrm{~m}, 3 \mathrm{H}), 3.84(\mathrm{~m}, 2 \mathrm{H}), 3.70(\mathrm{~s}, 3 \mathrm{H}) ;{ }^{13} \mathrm{C}$ NMR $(100$ $\mathrm{MHz}, \mathrm{DMSO}-d_{6} ; \delta$, ppm) $162.3\left({ }^{1} J_{\mathrm{CF}}=243.8 \mathrm{~Hz}\right), 160.0,155.1$, $145.8,142.4,137.8,136.7\left({ }^{6} J_{\mathrm{CF}}=5.1 \mathrm{~Hz}\right), 130.7\left({ }^{5} J_{\mathrm{CF}}=9.3 \mathrm{~Hz}\right)$, $130.6,128.6,128.1,127.8,127.7\left({ }^{4} J_{\mathrm{CF}}=9.6 \mathrm{~Hz}\right), 127.4,126.7$, $126.6,122.4,121.5,120.0,119.0,118.4,113.8\left({ }^{2} J_{\mathrm{CF}}=24.8 \mathrm{~Hz}\right)$, $113.5,110.4,105.7\left({ }^{3} J_{\mathrm{CF}}=20.1 \mathrm{~Hz}\right), 105.5,104.6,54.3,47.7,32.7$; IR $\left(\mathrm{KBr}, v, \mathrm{~cm}^{-1}\right) 3502,3047,1502,1422,1235,1099,1015,946$, 851, 733; HRMS (APCI-TOF) $\mathrm{m} / \mathrm{z}$ calcd for $\mathrm{C}_{27} \mathrm{H}_{19} \mathrm{FNO}[\mathrm{M}-\mathrm{H}]^{-}$ 392.1451; found 392.1450.

\section{8-(1-methyl-1H-indol-3-yl)-8-(p-tolyl)-7,8- dihydrocyclobuta[h]quinolin-5-ol \\ (3v)}

White solid, $80 \mathrm{mg}$, $68 \%$ yield; $\mathrm{mp} 162-164^{\circ} \mathrm{C} ;{ }^{1} \mathrm{H}$ NMR (400 $\left.\mathrm{MHz}, \mathrm{DMSO}-d_{6} ; \delta, \mathrm{ppm}\right) 10.56(\mathrm{~s}, 1 \mathrm{H}), 8.88(\mathrm{~m}, 1 \mathrm{H}), 8.60(\mathrm{~d}$, $J=8.4 \mathrm{~Hz}, 1 \mathrm{H}), 7.72(\mathrm{~d}, J=8.0 \mathrm{~Hz}, 2 \mathrm{H}), 7.41(\mathrm{dd}, J=8.4,4.2 \mathrm{~Hz}$, $1 \mathrm{H}), 7.32(\mathrm{~d}, J=8.0 \mathrm{~Hz}, 1 \mathrm{H}), 7.23(\mathrm{~d}, J=8.0 \mathrm{~Hz}, 1 \mathrm{H}), 7.07(\mathrm{~d}, J$ $=8.0 \mathrm{~Hz}, 3 \mathrm{H}), 7.01(\mathrm{~s}, 1 \mathrm{H}), 6.90(\mathrm{~s}, 1 \mathrm{H}), 6.85(\mathrm{~m}, 1 \mathrm{H}), 3.91(\mathrm{~m}$, $2 \mathrm{H}), 3.64(\mathrm{~s}, 3 \mathrm{H}), 2.23(\mathrm{~s}, 3 \mathrm{H}) ;{ }^{13} \mathrm{C}$ NMR (100 MHz, DMSO- $d_{6} ; \delta$, 
ppm) 155.0, 151.2, 145.1, 143.9, 137.5, 137.1, 135.0, 132.7, 128.8, $128.3,128.1,126.5,121.3,120.4,120.3,120.1,119.3,118.8,110.1$, 105.3, 55.2, 46.1, 32.6, 21.1; IR (KBr, $\left.v, \mathrm{~cm}^{-1}\right)$ 3545, 3067, 1552, $1421,1230,1129,1012,949,840,730$; HRMS (APCI-TOF) m/z calcd for $\mathrm{C}_{27} \mathrm{H}_{21} \mathrm{~N}_{2} \mathrm{O}[\mathrm{M}-\mathrm{H}]^{-}$389.1654; found 389.1657.

\section{1-(4-chlorophenyl)-1-(5-methoxy-1-methyl-1H-indol- 3-yl)-1,2-dihydrocyclobuta[a]naphthalen-4-ol (3w)}

White solid, $121 \mathrm{mg}, 92 \%$ yield; mp $166-168^{\circ} \mathrm{C} ;{ }^{1} \mathrm{H}$ NMR (400 $\mathrm{MHz}, \mathrm{DMSO}-d_{6}$; $\left.\delta, \mathrm{ppm}\right) 10.27(\mathrm{~s}, 1 \mathrm{H}), 8.27(\mathrm{~d}, J=8.0 \mathrm{~Hz}, 1 \mathrm{H})$, $7.48(\mathrm{~d}, J=8.0 \mathrm{~Hz}, 1 \mathrm{H}), 7.44-7.33(\mathrm{~m}, 6 \mathrm{H}), 7.27(\mathrm{~d}, J=8.8 \mathrm{~Hz}$, $1 \mathrm{H}), 6.88(\mathrm{~d}, J=10.8 \mathrm{~Hz}, 2 \mathrm{H}), 6.74(\mathrm{~m}, 1 \mathrm{H}), 6.24(\mathrm{~d}, J=2.0 \mathrm{~Hz}$, $1 \mathrm{H}), 3.81(\mathrm{~m}, 2 \mathrm{H}), 3.64(\mathrm{~s}, 3 \mathrm{H}), 3.49(\mathrm{~s}, 3 \mathrm{H}) ;{ }^{13} \mathrm{C} \mathrm{NMR}(100 \mathrm{MHz}$, DMSO- $d_{6} ; \delta$, ppm) $155.0,153.3,145.0,140.5,136.5,133.1,131.1$, $130.0,129.4,128.7,128.5,127.6,127.0,125.3,124.4,124.0,122.2$, $117.6,111.0,110.9,104.8,102.4,55.5,53.8,47.7,32.8$; IR (KBr, $v, \mathrm{~cm}^{-1}$ ) 3504, 2997, 1534, 1401, 1233, 1149, 1010, 942, 847, 736; HRMS (APCI-TOF) $\mathrm{m} / \mathrm{z}$ calcd for $\mathrm{C}_{28} \mathrm{H}_{21} \mathrm{NClO}_{2}[\mathrm{M}-\mathrm{H}]^{-}$ 438.1261; found 438.1267.

\section{1-(5-methoxy-1-methyl-1H-indol-3-yl)-1-(p-tolyl)-1,2- dihydrocyclobuta[a]naphthalen-4-ol}

(3x)

White solid, $107 \mathrm{mg}, 85 \%$ yield; mp $170-172^{\circ} \mathrm{C} ;{ }^{1} \mathrm{H}$ NMR (400 $\left.\mathrm{MHz}, \mathrm{DMSO}-d_{6} ; \delta, \mathrm{ppm}\right) 10.21(\mathrm{~s}, 1 \mathrm{H}), 8.25(\mathrm{~d}, J=8.0 \mathrm{~Hz}, 1 \mathrm{H})$, $7.49(\mathrm{~d}, J=8.0 \mathrm{~Hz}, 1 \mathrm{H}), 7.43-7.33(\mathrm{~m}, 2 \mathrm{H}), 7.29(\mathrm{~d}, J=8.0 \mathrm{~Hz}$, $2 \mathrm{H}), 7.25(\mathrm{~d}, J=8.8 \mathrm{~Hz}, 1 \mathrm{H}), 7.11(\mathrm{~d}, J=7.6 \mathrm{~Hz}, 2 \mathrm{H}), 6.88$ (s, $1 \mathrm{H}), 6.82(\mathrm{~s}, 1 \mathrm{H}), 6.72(\mathrm{~m}, 1 \mathrm{H}), 6.22(\mathrm{~d}, J=2.0 \mathrm{~Hz}, 1 \mathrm{H}), 3.79$ $(\mathrm{m}, 2 \mathrm{H}), 3.63(\mathrm{~s}, 3 \mathrm{H}), 3.47(\mathrm{~s}, 3 \mathrm{H}), 2.27(\mathrm{~s}, 3 \mathrm{H}) ;{ }^{13} \mathrm{C}$ NMR $(100$ $\left.\mathrm{MHz}, \mathrm{DMSO}-d_{6} ; \delta, \mathrm{ppm}\right) 154.8,153.1,143.0,140.5,137.1,135.3$, $133.1,130.2$, 129.1, 128.6, 127.5, 127.4, 127.1, 125.2, 124.4, 123.8, $122.4,118.4,110.8,110.7,104.9,102.6,55.5,54.1,47.7,32.8,21.1$; IR $\left(\mathrm{KBr}, v, \mathrm{~cm}^{-1}\right) 3487,3022,1520,1422,1200,1097,1012,944$, 833,725 ; HRMS (APCI-TOF) $\mathrm{m} / \mathrm{z}$ calcd for $\mathrm{C}_{29} \mathrm{H}_{24} \mathrm{NO}_{2}[\mathrm{M}-\mathrm{H}]^{-}$ 418.1808; found 418.1838 .

\section{1-(7-chloro-1-methyl-1H-indol-3-yl)-1-(p-tolyl)-1,2- dihydrocyclobuta[a]naphthalen-4-ol}

(3y)

White solid, $113 \mathrm{mg}, 89 \%$ yield; mp $163-165^{\circ} \mathrm{C} ;{ }^{1} \mathrm{H}$ NMR (400 $\left.\mathrm{MHz}, \mathrm{DMSO}-d_{6} ; \delta, \mathrm{ppm}\right) 10.24(\mathrm{~s}, 1 \mathrm{H}), 8.24(\mathrm{~d}, J=8.0 \mathrm{~Hz}$, $1 \mathrm{H}), 7.48(\mathrm{~d}, J=7.6 \mathrm{~Hz}, 1 \mathrm{H}), 7.38(\mathrm{~m}, 2 \mathrm{H}), 7.23(\mathrm{~d}, J=8.0 \mathrm{~Hz}$, $2 \mathrm{H}), 7.06(\mathrm{~d}, J=8.4 \mathrm{~Hz}, 2 \mathrm{H}), 6.93(\mathrm{~s}, 1 \mathrm{H}), 6.88-6.76(\mathrm{~m}, 4 \mathrm{H})$, 3.99 (s, 3H), $3.78(\mathrm{~s}, 2 \mathrm{H}), 2.24(\mathrm{~s}, 3 \mathrm{H}) ;{ }^{13} \mathrm{C} \mathrm{NMR}(100 \mathrm{MHz}$, DMSO- $d_{6} ; \delta$, ppm) $154.8,142.5,140.2,136.9,135.5,132.6,131.2$, $130.2,129.9,129.2,127.4,127.3,125.2,124.4,123.9,123.0,122.2$, $120.1,119.6,119.3,116.5,104.9,53.6,47.6,36.5,21.0$; IR (KBr, $\left.v, \mathrm{~cm}^{-1}\right) 3505,3022,1524,1400,1209,1082,1012,945,833$, 719; HRMS (APCI-TOF) $\mathrm{m} / \mathrm{z}$ calcd for $\mathrm{C}_{28} \mathrm{H}_{21} \mathrm{ClNO}[\mathrm{M}-\mathrm{H}]^{-}$ 422.1312; found 422.1304 .

\section{1-(1,7-dimethyl-1H-indol-3-yl)-1-(p-tolyl)-1,2- dihydrocyclobuta[a]naphthalen-4-ol}

(3z)

White solid, $93 \mathrm{mg}, 77 \%$ yield; mp $165-167^{\circ} \mathrm{C} ;{ }^{1} \mathrm{H}$ NMR (400 MHz, DMSO- $d_{6}$; $\delta$, ppm) $10.17(\mathrm{~s}, 1 \mathrm{H}), 8.23$ (d, $\left.J=8.0 \mathrm{~Hz}, 1 \mathrm{H}\right)$, $7.48(\mathrm{~d}, J=8.0 \mathrm{~Hz}, 1 \mathrm{H}), 7.37(\mathrm{~m}, 2 \mathrm{H}), 7.22(\mathrm{~d}, J=8.0 \mathrm{~Hz}, 2 \mathrm{H})$, $7.06(\mathrm{~d}, J=8.0 \mathrm{~Hz}, 2 \mathrm{H}), 6.85(\mathrm{~s}, 1 \mathrm{H}), 6.79-6.64(\mathrm{~m}, 4 \mathrm{H}), 3.93(\mathrm{~s}$, $3 \mathrm{H}), 3.77$ (s, 2H), 2.68 (s, 3H), 2.25 (s, 3H). ${ }^{13} \mathrm{C} \mathrm{NMR}(100 \mathrm{MHz}$, DMSO- $d_{6} ; \delta$, ppm) 154.6, 143.0, 140.3, 137.4, 136.3, 135.3, 129.9, 129.6, 129.1, 128.0, 127.3, 125.2, 124.3, 124.0, 123.8, 122.4, 121.8, 119.1, 118.5, 118.4(5), 118.3(9), 105.0, 53.8, 47.6, 36.6, 21.1, 19.7; IR $\left(\mathrm{KBr}, v, \mathrm{~cm}^{-1}\right) 3468,3056,1480,1424,1203,1095,1010,958$, 844, 722; HRMS (APCI-TOF) m/z calcd for $\mathrm{C}_{29} \mathrm{H}_{24} \mathrm{NO}[\mathrm{M}-\mathrm{H}]^{-}$ 402.1858; found 402.1844 .

\section{1-(6-methoxy-1-methyl-1H-indol-3-yl)-1-(p-tolyl)-1,2- dihydrocyclobuta[a]naphthalen-4-ol \\ (3aa)}

White solid, $96 \mathrm{mg}, 76 \%$ yield; mp $171-173^{\circ} \mathrm{C}$; ${ }^{1} \mathrm{H}$ NMR (400 $\mathrm{MHz}, \mathrm{DMSO}-d_{6} ; \delta$, ppm) $10.18(\mathrm{~s}, 1 \mathrm{H}), 8.23(\mathrm{~d}, J=8.0 \mathrm{~Hz}, 1 \mathrm{H})$, $7.48(\mathrm{~d}, J=8.0 \mathrm{~Hz}, 1 \mathrm{H}), 7.41-7.32(\mathrm{~m}, 2 \mathrm{H}), 7.26(\mathrm{~d}, J=8.0 \mathrm{~Hz}$, $2 \mathrm{H}), 7.08(\mathrm{~d}, J=8.0 \mathrm{~Hz}, 2 \mathrm{H}), 6.89(\mathrm{~d}, J=2.0 \mathrm{~Hz}, 1 \mathrm{H}), 6.85(\mathrm{~s}$, $1 \mathrm{H}), 6.70(\mathrm{~d}, J=10.4 \mathrm{~Hz}, 2 \mathrm{H}), 6.48(\mathrm{~m}, 1 \mathrm{H}), 3.81(\mathrm{~m}, 2 \mathrm{H}), 3.75(\mathrm{~s}$, $3 \mathrm{H}), 3.63(\mathrm{~s}, 3 \mathrm{H}), 2.26(\mathrm{~s}, 3 \mathrm{H}) ;{ }^{13} \mathrm{C} \mathrm{NMR}\left(100 \mathrm{MHz}, \mathrm{DMSO}-d_{6} ; \delta\right.$, ppm) 156.0, 154.6, 143.1, 140.3, 138.5, 137.3, 135.3, 130.0, 129.1, $127.4,127.3,126.8,125.2,124.3,123.8,122.4,121.1,120.7,119.1$, 109.0, 105.0, 93.6, 55.7, 54.0, 47.8, 32.7, 21.1; IR (KBr, v, $\left.\mathrm{cm}^{-1}\right)$ 3487, 3020, 1521, 1420, 1212, 1091, 1011, 944, 836, 729; HRMS (APCI-TOF) $\mathrm{m} / \mathrm{z}$ calcd for $\mathrm{C}_{29} \mathrm{H}_{24} \mathrm{NO}_{2}[\mathrm{M}-\mathrm{H}]^{-} 418.1808$; found 418.1822 .

\section{1-(5-bromo-1-methyl-1H-indol-3-yl)-1-(p-tolyl)-1,2- dihydrocyclobuta[a]naphthalen-4-ol \\ (3bb)}

White solid, $121 \mathrm{mg}, 76 \%$ yield; $\mathrm{mp} 175-177^{\circ} \mathrm{C}$; ${ }^{1} \mathrm{H}$ NMR (400 MHz, DMSO- $\left.d_{6} ; \delta, \mathrm{ppm}\right) 10.22(\mathrm{~s}, 1 \mathrm{H}), 8.25(\mathrm{~d}, J=8.0 \mathrm{~Hz}, 1 \mathrm{H})$, $7.46(\mathrm{~d}, J=8.0 \mathrm{~Hz}, 1 \mathrm{H}), 7.40(\mathrm{~m}, 3 \mathrm{H}), 7.25(\mathrm{~d}, J=8.4 \mathrm{~Hz}, 2 \mathrm{H})$, $7.20(\mathrm{~d}, J=8.0 \mathrm{~Hz}, 1 \mathrm{H}), 7.10(\mathrm{~d}, J=8.0 \mathrm{~Hz}, 2 \mathrm{H}), 6.95(\mathrm{~s}, 1 \mathrm{H})$, $6.93(\mathrm{~s}, 1 \mathrm{H}), 6.86(\mathrm{~s}, 1 \mathrm{H}), 3.84-3.74(\mathrm{~m}, 2 \mathrm{H}), 3.69(\mathrm{~s}, 3 \mathrm{H}), 2.27(\mathrm{~s}$, $3 \mathrm{H}) ;{ }^{13} \mathrm{C}$ NMR $\left(100 \mathrm{MHz}, \mathrm{DMSO}-d_{6} ; \delta\right.$, ppm) $154.8,142.5,140.3$, $136.8,136.5,135.5,129.9,129.6,129.2,128.4,127.5,127.3,125.2$, $124.4,124.0,122.2,118.8,112.6,111.6,104.9,53.7,47.8,32.9$, 21.1; IR (KBr, v, $\left.\mathrm{cm}^{-1}\right)$ 3531, 3050, 1522, 1404, 1242, 1121, 1017, 945, 836, 720; HRMS (APCI-TOF) $\mathrm{m} / \mathrm{z}$ calcd for $\mathrm{C}_{28} \mathrm{H}_{21} \mathrm{BrNO}$ $[\mathrm{M}-\mathrm{H}]^{-}$466.0807; found 466.0815.

\section{1-(1H-indol-3-yl)-1-(p-tolyl)-1,2- dihydrocyclobuta[a]naphthalen-4-ol} (3cc)

White solid, $88 \mathrm{mg}, 78 \%$ yield; $\mathrm{mp} 156-158^{\circ} \mathrm{C}$; ${ }^{1} \mathrm{H}$ NMR (400 $\left.\mathrm{MHz}, \mathrm{DMSO}-d_{6} ; \delta, \mathrm{ppm}\right) 10.86(\mathrm{~s}, 1 \mathrm{H}), 10.20(\mathrm{~s}, 1 \mathrm{H}), 8.26(\mathrm{~d}$, $J=8.4 \mathrm{~Hz}, 1 \mathrm{H}), 7.52(\mathrm{~d}, J=8.0 \mathrm{~Hz}, 1 \mathrm{H}), 7.38(\mathrm{~m}, 3 \mathrm{H}), 7.27(\mathrm{~d}$, $J=8.4 \mathrm{~Hz}, 2 \mathrm{H}), 7.08$ (d, $J=8.0 \mathrm{~Hz}, 2 \mathrm{H}), 7.02(\mathrm{~m}, 1 \mathrm{H}), 6.96-6.88$ $(\mathrm{m}, 3 \mathrm{H}), 6.81(\mathrm{~m}, 1 \mathrm{H}), 3.82(\mathrm{~m}, 2 \mathrm{H}), 2.25(\mathrm{~s}, 3 \mathrm{H}) ;{ }^{13} \mathrm{C}$ NMR $(100$ MHz, DMSO- $d_{6} ; \delta$, ppm) 154.6, 143.2, 140.3, 137.4, 137.4, 135.3, $130.0,129.1,127.4,127.3,126.6,125.2,124.4,123.8,123.7,122.4$, $122.0,121.3,120.0,119.7,118.8,112.1,105.0,54.1,47.6,21.1$; IR $\left(\mathrm{KBr}, v, \mathrm{~cm}^{-1}\right) 3501,3408,3051,1520,1402,1240,1118,1010$, 943, 816, 723; HRMS (APCI-TOF) $\mathrm{m} / \mathrm{z}$ calcd for $\mathrm{C}_{27} \mathrm{H}_{20} \mathrm{NO}$ $[\mathrm{M}-\mathrm{H}]^{-}$374.1545; found 374.1529 . 


\section{1-(6-methyl-1H-indol-3-yl)-1-phenyl-1,2- dihydrocyclobuta[a]naphthalen-4-ol \\ (3dd)}

White solid, $91 \mathrm{mg}, 81 \%$ yield; mp $172-174{ }^{\circ} \mathrm{C} ;{ }^{1} \mathrm{H}$ NMR (400 $\left.\mathrm{MHz}, \mathrm{DMSO}-d_{6} ; \delta, \mathrm{ppm}\right) 10.72(\mathrm{~s}, 1 \mathrm{H}), 10.18(\mathrm{~s}, 1 \mathrm{H}), 8.25$ $(\mathrm{d}, J=8.4 \mathrm{~Hz}, 1 \mathrm{H}), 7.52(\mathrm{~d}, J=8.0 \mathrm{~Hz}, 1 \mathrm{H}), 7.44-7.34(\mathrm{~m}$, $4 \mathrm{H}), 7.26(\mathrm{~m}, 3 \mathrm{H}), 7.18(\mathrm{~m}, 1 \mathrm{H}), 6.86(\mathrm{~d}, J=6.8 \mathrm{~Hz}, 3 \mathrm{H})$, $6.79(\mathrm{~s}, 1 \mathrm{H}), 3.83(\mathrm{~s}, 2 \mathrm{H}), 2.22(\mathrm{~s}, 3 \mathrm{H}) ;{ }^{13} \mathrm{C} \mathrm{NMR}(100 \mathrm{MHz}$, DMSO- $d_{6} ; \delta$, ppm) $154.6,146.2,140.4,137.5,135.7,129.9,128.5$, $127.3,127.1,126.9,126.3,125.2,124.4,123.8,123.0,122.4,119.6$, 119.0, 111.9, 105.0, 54.2, 47.5, 21.9; IR (KBr, v, $\left.\mathrm{cm}^{-1}\right) 3518$, 3401, 3050, 1570, 1392, 1242, 1110, 1023, 941, 826, 721; HRMS (APCI-TOF) $\mathrm{m} / \mathrm{z}$ calcd for $\mathrm{C}_{27} \mathrm{H}_{20} \mathrm{NO}[\mathrm{M}-\mathrm{H}]^{-}$374.1545; found 374.1553.

\section{X-Ray Structure of Product 3a (CCDC 1867087)}

The crystal of compound 3a belongs to Triclinic, space group $P$-1 with $a=8.5599(7) \AA$, $b=12.1512(11) \AA, c=12.5112(12)$ $\AA, \alpha=100.943(2)^{\circ}, \beta=94.2510(10)^{\circ}, \gamma=106.823(3)^{\circ}, V$ $=1211.40(19) \AA^{3}, M r=433.53, Z=2, D c=1.743 \mathrm{~g} / \mathrm{cm}^{3}$,

\section{REFERENCES}

Aidhen, I. S., and Ahuja, J. R. (1992). A novel synthesis of benzocyclobutenones. Tetrahedron Lett. 33, 5431-5432. doi: 10.1016/S0040-4039(00)79 113-1

Anastas, P. T., and Warner, J. C. (1998). Green Chemistry: Theory and Practice. New York, NY: Oxford Science Publications.

Andersen, N. G., Maddaford, S. P., and Keay, B. A. (1996). Synthesis of functionalized naphthalenes from substituted 1methoxybenzocyclobutenes. J. Org. Chem. 61, 2885-2887. doi: 10.1021/jo95 $1978 \mathrm{~V}$

Banert, K., and Plefka, O. (2011). Synthesis with perfect atom economy: Generation of diazo ketones by 1,3-dipolar cycloaddition of nitrous oxide at cyclic alkynes under mild conditions. Angew. Chem. Int. Ed. 50, 6171-6174. doi: 10.1002/anie.201101326

Barluenga, J., Rodriguez, F., and Fananas, F. J. (2009). Recent advances in the synthesis of indole and quinoline derivatives through cascade reactions. Chem. Asian J. 4, 1036-1048. doi: 10.1002/asia.2009 00018

Beak, P., and Selling, G. W. (1989). Displacements at the nitrogen of lithioalkoxylamides by organometallic reagents. J. Org. Chem. 54, 5574-5580. doi: 10.1021/jo00284a034

Bihani, M., Bora, P. P., Bez, G., and Askari, H. (2013). Amberlyst A21 catalyzed chromatography-free method for multicomponent synthesis of dihydropyrano [2,3-c] pyrazoles in ethanol. ACS Sustainable Chem. Eng. 1, 440-447. doi: $10.1021 / \mathrm{sc} 300173 \mathrm{z}$

Bilyard, K. G., Garratt, P. J., Underwood, A. J., and Zahler, R. (1979). Vicinal diester dianions. Annelation with allylic and benzylic $\alpha \omega$-dihalides. Synthesis of tricyclobuta[1,2:3,4:6,7]naphthalene. Tetrahedron Lett. 20, 1815-1818. doi: 10.1016/S0040-4039(01)86226-2

Birch, A. J., Brown, J. M., and Stansfield, F.,(1964). Reactions of cyclohexadienes. IV. Some transformations of bisdihalocarbene adducts. J. Chem. Soc. 5343-5348. doi: 10.1039/jr9640005343

Bradsher, C. K., and Hunt, D. A. (1981). Novel synthesis of benzo[1,2:4,5] dicyclobutene via a dual Parham cyclialkylation. J. Org. Chem. 46, 4608-4610. doi: 10.1021/jo00335a072

Brauch, S., Van Berkel, S.-S., and Westermann, B. (2013). Higher-order multicomponent reactions: beyond four reactants. Chem. Soc. Rev. 42, 4948-4962. doi: 10.1039/c3cs35505e
$\mu(\mathrm{MoK} \alpha)=0.074 \mathrm{~mm}^{-1}, F(000)=460$, the final $R=0.0495$ and $w R=0.1118$.

\section{AUTHOR CONTRIBUTIONS}

$\mathrm{HL}, \mathrm{BJ}$, and GL designed the project. HL performed the experiments. HL, W-JH, and S-JT analyzed the data. HL, BJ, and GL wrote the manuscript.

\section{FUNDING}

We are grateful for financial support from the NSFC (Nos. 21332005, 21472071 and 21871112), the Outstanding Youth Fund of JSNU (YQ2015003) and Robert A. Welch Foundation (D-1361, USA).

\section{SUPPLEMENTARY MATERIAL}

The Supplementary Material for this article can be found online at: https://www.frontiersin.org/articles/10.3389/fchem. 2018.00599/full\#supplementary-material

Bruckmann, A., Krebs, A., and Bolm, C. (2008). Organocatalytic reactions Effects of ball milling, microwave and ultrasound irradiation. Green Chem. 10, 1131-1141. doi: 10.1039/b812536h

Buchwald, S. L., Lucas, E. A., and Dewan, J. C. (1987a). Synthesis, structure, and reactions of a zirconocene-benzdiyne complex. J. Am. Chem, Soc. 109, 4396-4397. doi: 10.1021/ja00248a046

Buchwald, S. L., Watson, B. T., Lum, R. T., and Nugent, W. A. (1987b). A general method for the preparation of zirconocene complexes of substituted benzynes: in situ generation, coupling reactions, and use in the synthesis of polyfunctionalized aromatic compounds. J. Am. Chem. Soc. 109, 7137-7141. doi: $10.1021 /$ ja00257a038

Buckland, S. J., Halton, B., Mei, Q., and Stang, P. J. (1987). Studies in the cycloproparene series: reactions of alkylidenecycloproparenes with electrophiles. Aust. J. Chem. 40, 1375-1387. doi: 10.1071/CH9871375

Bunnett, J. F., and Skorcz, J. A. (1962). Homocyclic ring closures via benzyne intermediates. A new synthesis of 1-substituted benzocyclobutenes. J. Org. Chem. 27, 3836-3843. doi: 10.1021/jo01058a019

Cava, M. P., and Napier, D. R. (1956). Benzocyclobutene and benzocyclobutadiene dimer. J. Am. Chem. Soc. 78:500. doi: 10.1021/ja01583a071

Chen, K., Liu, S., Wang, D., Hao, W.-J., Zhou, P., Tu, S.-J., et al. (2017). Silver/scandium-cocatalyzed bicyclization of $\beta$-Alkynyl ketones leading to benzo $[c]$ xanthenes and naphtho[1,2-b]benzofurans. J. Org. Chem. 82, 11524-11530. doi: 10.1021/acs.joc.7b02134

Chou, C.-H., Wu, C.-C., and Chen, W.-K. (1995). Synthesis of Pyrido $[b]$ cyclobuten-5-one and 1-azafulvenallene by flash vacuum pyrolysis of 3-chloroformyl-2-methylpyridine. Tetrahedron Lett. 36:5065. doi: 10.1016/0040-4039(95)00940-E

Christophe, S., Kuehm-Caubere, C., Renard, P., Pfeiffer, B., and Caubere, P. (1998). Design of new anticancer drugs. II. Easy arynic access to benzocyclobutacarbazoles, a new family of antitumor agents. Tetrahedron Lett. 39, 7284-7286. doi: 10.1016/S0040-4039(98)02186-8

Craig, D., Robson, M. J., and Shaw, S. J. (1998). Traceless linkers for solid-phase synthesis. Homo-and hetero-Diels-Alder reactions of o-quinodimethanes. Synlett 1998, 1381-1383. doi: 10.1055/s-1998-1968

D’Andrea, S. V., Freeman, J. P., and Szmuszkovicz, J. (1990). Diels-Alder cycloaddition reactions of isobenzofuran and o-quinodimethane with 1,2 diheteroethylenes. J. Org. Chem. 55, 4356-4358. doi: 10.1021/jo00301a028

Dömling, A., Wang, W., and Wang, K. (2012). Chemistry and biology of multicomponent reactions. Chem. Rev. 112, 3083-3135. doi: 10.1021/cr100233r 
Ezcurra, J. E., and Moore, H. W. (1993). Synthesis of o-quinodimethanes and benzocyclobutenes from dimethyl squarate. Tetrahedron Lett. 34, 6177-6180. doi: 10.1016/S0040-4039(00)73703-8

Fuerstner, A. (2009). Gold and platinum catalysis-a convenient tool for generating molecular complexity. Chem. Soc. Rev. 38, 3208-3221. doi: 10.1039/b816696j

Funk, R. L., and Vollhardt, K. P. C. (1977). A cobalt-catalyzed steroid synthesis. J. Am. Chem. Soc. 99, 5483-5484. doi: 10.1021/ja00458a044

Funk, R. L., and Vollhardt, K. P. C. (1979). The cobalt way to dl-estrone, a highly regiospecific functionalization of 2, 3-bis (trimethylsilyl) estratrien-17-one. J. Am. Chem. Soc. 101, 215-217. doi: 10.1021/ja00495a035

Funk, R. L., and Vollhardt, K. P. C. (1980). Cooligomerizations of 3-substituted 1, 5-hexadiynes with bis(trimethylsilyl)acetylene catalyzed by cobalt. A general synthesis of tricyclic ring systems from acyclic precursors. J. Am. Chem. Soc. 102, 5245-5253. doi: 10.1021/ja00536a022

Garratt, P. J., and Nicolaides, D. N. (1972). 3-Thiabicyclo[3.2.0]hepta-1, 4-diene. J. Chem. Soc. Chem. Commun. 1014-1015. doi: 10.1039/c39720001014

Garratt, P. J., and Nicolaides, D. N. (1974). Synthesis and some reactions of 3thiabicyclo [3.2.0] hepta-1, 4-diene. Case for revival of the Mills-Nixon effect. J. Org. Chem. 39, 2222-2224. doi: 10.1021/jo00929a018

Gowland, B. D., and Durst, T. (1979). Benzocyclobutyl phenyl sulfone. An evaluation of its potential as a precursor to substituted benzocyclobutenes and ortho-quinodimethanes. Can. J. Chem. 57, 1462-1467. doi: 10.1139/v79-239

Gray, R., Harruff, L. G., Krymowski, J., Peterson, J., and Boekelheide, V. (1978), Syntheses of benzo[1,2:4,5]dicyclobutene derivatives. J. Am. Chem. Soc. 100, 2892-2893. doi: 10.1021/ja00477a054

Grieco, P. A., Takigawa, T., and Schillinger, W. J. (1980). Bicyclo[2.2.1]heptanes as intermediates in the synthesis of steroids. Total synthesis of estrone. J. Org. Chem. 45, 2247-2251. doi: 10.1021/jo01299a042

Haibach, M. C., Deb, I., De, C. K., and Seidel, D. (2011). Redox-neutral indole annulation cascades. J. Am. Chem. Soc. 133, 2100-2103. doi: 10.1021/ja11 $0713 \mathrm{k}$

Hickman, D. N., Wallace, T. W., and Wardleworth, J. M. (1991). The preparation of $\alpha$-tetralones from benzocyclobutenones via sequential thermal electrocyclic reactions. Tetrahedron Lett. 32, 819-822. doi: 10.1016/S0040-4039(00) 74896-9

Hoffmann, N., and Pete, J.-P. (1996). Acid catalyzed intramolecular photochemical reactions of 3-alkenyloxyphenols. Tetrahedron Lett. 37, 2027-2030. doi: 10.1016/0040-4039(96)00190-6

Huang, M.-H., Hao, W.-J., and Jiang, B. (2018a). Radical enabled bicyclization and annulation with 1,n-bifunctionalization: recent advances. Chem. Asian J. 13, 2958-2977. doi: 10.1002/asia.201801119

Huang, M.-H., Hao, W.-J., Li, G., Tu, S.-J., and Jiang, B. (2018b). Recent advances in radical transformations of internal alkynes. Chem. Commun. 54, 10791-10811. doi: 10.1039/C8CC04618B

Huang, M.-H., Zhu, Y.-L., Hao, W.-J., Wang, A.-F., Wang, D.-C., Liu, F., et al. (2017). Visible-light photocatalytic bicyclization of 1,7-enynes toward functionalized sulfone-containing benzo[a]fluoren-5-ones. Adv. Synth. Catal. 359, 2229-2234. doi: 10.1002/adsc.201700124

Inanaga, J., Sugimoto, Y., and Hanamoto, T. (1992). A novel method for the generation of 2,3-naphthoquinodimethanes utilizing samarium (II) iodide-promoted allene synthesis. Tetrahedron Lett. 33, 7035-7038. doi: 10.1016/S0040-4039(00)60926-7

Iskander, G. M., and Stansfield, F. (1965). 4-Hydroxybenzocyclobuten-I-one and its derivatives. J. Chem. Soc. 1390-1392. doi: 10.1039/jr9650001390

Jia, S., Su, S., Li, C., Jia, X., and Li, J. (2014). Multicomponent cascade cycloaddition involving tropone, allenoate, and isocyanide: a rapid access to a 7, 6, 5-fused tricyclic skeleton. Org. Lett. 16, 5604-5607. doi: 10.1021/ol502656g

Jiang, B., Rajale, T., Wever, W., Tu, S.-J., and Li, G. (2010). Multicomponent reactions for the synthesis of heterocycles. Chem. Asian J. 5, 2318-2335. doi: 10.1002/asia.201000310

Jones, A. C., May, J. A., Sarpong, R., and Stoltz, B. M. (2010). Toward a symphony of reactivity: cascades involving catalysis and sigmatropic rearrangements. Angew. Chem. Int. Ed. 53, 2556-2591. doi: 10.1002/anie.2013 02572

Kagabu, S., and Saito, K. (1988). Dihalocarbene addition to benzocyclopropene. Tetrahedron Lett. 29, 675-676. doi: 10.1016/S0040-4039(00)80181-1

Kanao, Y., Iyoda, M., and Oda, M. (1983). The effect of cyclobutene ring annulation on p-benzoquinone: synthesis and properties of 1,2,3,4,5,6-hexahydrobenzo[1,2:4,5]dicyclobutene-3,6dione([2,3:5,6]dicyclobuta-p-benzoquinone). Tetrahedron Lett. 24, 1727-1730. doi: 10.1016/S0040-4039(00)81755-4

Kaneko, C., and Naito, T. (1979). Intermolecular photochemical cycloaddition of 4-methoxy-2-quinolone with olefins: a regioselective synthesis of 5substituted cyclobuta[c]-2-quinolones. Chem. Pharm. Bull. 27, 2254-2256. doi: $10.1248 / \mathrm{cpb} .27 .2254$

Kaneko, C., Naito, T., Momose, Y., Fujii, H., Nakayama, N., and Koizumi, I. (1982). Cycloadditions in syntheses. VIII. Synthesis of 1,2-dihydrocyclobuta[c]pyridine and-quinoline and their 3-substituted derivatives. Chem. Pharm. Bull. 30, 519-525. doi: $10.1248 / \mathrm{cpb} .30 .519$

Koopmanschap, G., Ruijter, E., and Orru, R. V. A. (2014). Isocyanide-based multicomponent reactions towards cyclic constrained peptidomimetics. J. Org. Chem. 10, 544-598. doi: 10.3762/bjoc. 10.50

Kotha, S., Goyal, D., and Chavan, A. S. (2013). Diversity-oriented approaches to unusual $\alpha$-amino acids and peptides: step economy, atom economy, redox economy, and beyond. J. Org. Chem. 78, 12288-12313. doi: 10.1021/jo4020722

Krohn, K., Carboo, D., and Puttfarcker, U. (1978). 1-Phenyl-1,2-dihydrobenzazetes from N-phenylbenzylamines by aryne cyclization. Liebigs Ann. Chem. 1978, 608-616. doi: 10.1002/jlac.197819780411

Lenihan, B. D., and Shechter, H. (1994). Substituted $o$-[(Trimethylsilyl) methyl] benzyl p-tolyl sulfones: practical reagents for preparing cycloadducts of o-quinodimethanes. Tetrahedron Lett. 35, 7505-7508. doi: 10.1016/S0040-4039(00)78329-8

Lenihan, B. D., and Shechter, H. (1998). Chemistry of Conversions of [o-[1Halo-1-( $p$-tolylsulfonyl)alkyl]benzyl] trimethylsilanes to $o$-Quinodimethanes and Benzocyclobutenes. J. Org. Chem. 63, 2086-2093. doi: 10.1021/jo9705935

Li, H., Hao, W.-J., Wang, M., Qin, X., Tu, S.-J., Zhou, P., et al. (2018a). Catalytic double $[2+2]$ cycloaddition relay enabled $\mathrm{C}-\mathrm{C}$ triple bond cleavage of Yne-Allenones. Org. Lett. 20, 4362-4366. doi: 10.1021/acs.orglett.8b01841

Li, H., Zhou, P., Xie, F., Hu, J.-Q., Yang, S.-Z., Wang, Y.-J., et al. (2018b). $\mathrm{I}_{2}$-Mediated iodobenzannulation of yne-allenones toward 1-naphthols and their synthetic application, J. Org. Chem. 83, 13335-13343. doi: 10.1021 /acs.joc. 8 b02108

Li, X., Li, H., Song, W., Tseng, P.-S., Liu, L., Guzei, I. A., et al. (2015). Divergent reactivity of rhodium (I) carbenes derived from indole annulations. Angew. Chem. Int. Ed. 54, 12905-12908. doi: 10.1002/anie.201505329

Liu, C., Zhou, L., Huang, W., Wang, M., and Gu, Y. (2016). Synthesis of dihydrocarbazoles via $(4+2)$ annulation of donor-acceptor cyclopropanes to indoles. Tetrahedron 72, 563-570. doi: 10.1016/j.tet.2015.12.023

Liu, F., Wang, J.-Y., Zhou, P., Li, G., Hao, W.-J., Tu, S.-J., et al. (2017a). Merging $[2+2]$ cycloaddition with radical 1,4-addition: metal-free access to functionalized cyclobuta[a]naphthalen-4-ols. Angew. Chem. Int. Ed. 56, 15570-15574. doi: 10.1002/anie.201707615

Liu, S., Chen, K., Lan, X.-C., Hao, W.-J., Li, G., Tu, S.-J., et al. (2017b). Synergistic silver/scandium catalysis for divergent synthesis of skeletally diverse chromene derivatives. Chem. Commun. 53, 10692-10695. doi: 10.1039/C7CC05563C

Liu, S., Lan, X.-C., Chen, K., Hao, W.-J., Li, G., Tu, S.-J., et al. (2017c). $\mathrm{Ag} / \mathrm{Br} ø n s t e d$ Acid Co-Catalyzed Spiroketalization of $\beta$-Alkynyl Ketones toward Spiro [chromane-2, $1^{\prime}$-isochromene] Derivatives. Org. Lett. 19, 3831-3834. doi: 10.1021/acs.orglett.7b01705

Markgraf, J. H., Katt, R. J., Scott, W. L., and Shefrin, R. N. (1969). Strained heterocyclic systems. IV. 1, 2-Dihydrocyclobuta [b] quinoline and derivatives. J. Org. Chem. 34, 4131-4133. doi: 10.1021/jo01264a081

Martins, M. A. P., Frizzo, C. P., Moreira, D. N., Buriol, L., and Machado, P. (2009). Solvent-free heterocyclic synthesis. Chem. Rev. 109, 4140-4182. doi: $10.1021 /$ cr9001098

McNichols, A. T., and Stang, P. J. (1992). An improved synthesis of cyclopropa[4,5]benzocyclobutene. Synlett 1992, 971-972. doi: $10.1055 / \mathrm{s}-1992-21549$

Mehta, G., and Kotha, S. (2001). Recent chemistry of benzocyclobutenes. Tetrahedron 57, 625-659. doi: 10.1016/S0040-4020(00)00958-3

Michellys, P.-Y., Maurin, P., Toupet, L., Pellissier, H., and Santelli, M. (2001). A very short synthesis of steroids from 1, 3-butadiene and benzocyclobutenes. J. Org. Chem. 66, 115-122. doi: 10.1021/jo001106f

Müller, P., Bernardinelli, G., Jacquier, Y., and Ricca, A. (1989). Tricarbonylchromium complexes of benzannelated cycloproparenes. Helv. Chim. Acta 72, 1618-1626. doi: 10.1002/hlca.19890720722 
Neckers, D. C., and Wagenaar, F. L. (1981). Synthesis of 3, 4-benzo2-thiabicyclo[3.2.0]hepta-1,3-diene. J. Org. Chem. 46, 3939-3940. doi: 10.1021/jo00332a046

Nemoto, H., Satoh, A., Fukumoto, K., and Kabuto, C. (1995). A rapid access to both enantiomers of 1,2,3,4-tetranor B-trienic 18,18,18-trifluorosteroids. The first enantiocontrolled total synthesis of 18,18,18-trifluorosteroids. J. Org. Chem. 60, 594-600. doi: 10.1021/jo00108a022

Ozaki, K., Matsuoka, W., Ito, H., and Itami, K. (2017). Annulative $\pi$-extension (APEX) of heteroarenes with dibenzosiloles and dibenzogermoles by palladium/o-chloranil catalysis. Org. Lett. 19, 1930-1933. doi: 10.1021/acs.orglett.7b00684

Parham, W. E., Jones, L. D., and Sayed, Y. A. (1976). Selective halogenlithium exchange in bromophenylalkyl halides. J. Org. Chem. 41, 1184-1186. doi: 10.1021/j000869a022

Peter, K., and Vollhardt, C. (1977). Transition-metal-catalyzed acetylene cyclizations in organic synthesis. Acc. Chem. Res. 10, 1-8. doi: 10.1021/ar50109a001

Peter, K., and Vollhardt, C. (1984). Cobalt-mediated [2+2+2]-cycloadditions: a maturing synthetic strategy [new synthetic methods (43)]. Angew. Chem. Int. Ed. 23, 539-556. doi: 10.1002/anie.198405393

Rotstein, B.-H., Zaretsky, S., Rai, V., and Yudin, A.-K. (2014). Small heterocycles in multicomponent reactions. Chem. Rev. 114, 8323-9359. doi: $10.1021 / \mathrm{cr} 400615 \mathrm{v}$

Sadana, A. K., Saini, R. K., and Billups, W. E. (2003). Cyclobutarenes and related compounds. Chem. Rev. 103, 1539-1602. doi: 10.1021/cr010022j

Sato, M., Suzuki, T., Morisawa, H., Fujita, S., Inukai, N., and Kaneko, C. (1987). Cycloadditions in syntheses. XXXV. 2-Cyano-1,2dihydrocyclobuta $[a]$ naphthalene and Its derivatives: synthesis and reaction with olefins. Chem. Pharm. Bull. 35, 3647-3657. doi: 10.1248/cpb.35.3647

Schirch, P. F. T., Boekelheide, V., and Cyclophane, M. (1979). J. Am. Chem. Soc. 101, 3125-3126. doi: 10.1021/ja00505a052

Sekine, Y., Brown, M., and Boekelheide, V. (1979). [2.2.2.2.2.2](1,2,3,4,5,6) Cyclophane: superphane. J. Am. Chem. Soc. 101, 3126-3127. doi: $10.1021 / \mathrm{ja} 00505 \mathrm{a} 053$

Sha, H.-K., Liu, F., Lu, J., Liu, Z.-Q., Hao, W.-J., Tang, J.-L., et al. (2018a). Metal-free benzannulation of yne-allenone esters for atom-economic synthesis of functionalized 1-naphthols. Green Chem. 20, 3476-3485 doi: 10.1039/C8GC01034J

Sha, H.-K., Xu, T., Liu, F., Tang, B.-Z., Hao, W.-J., Tu, S.-J., et al. (2018b). Metal-free naphthannulation reactions of yne-allenone esters for accessing polycyclic aromatic hydrocarbons. Chem. Commun. 54, 10415-10418. doi: $10.1039 / C 8 C C 05018$ J

Shimada, S., Osoda, K., and Narasaka, K. (1993). Boron compound as a trapping reagent of $\alpha$-hydroxy o-quinodimethanes in the diels-alder reaction. Bull. Chem. Soc. Jpn. 66, 1254-1257. doi: 10.1246/bcsj.66.1254

$\mathrm{Su}, \mathrm{S}$., Li, C., Jia, X., and Li, J. (2014). Isocyanide-based multicomponent reactions: concise synthesis of spirocyclic oxindoles with molecular complexity by using a [1,5]-hydrogen shift as the key step. Chem. Eur. J. 20, 5905-5909. doi: $10.1002 /$ chem. 201402576

Sugimoto, K., and Matsuya, Y. (2017). Recent applications of gold-catalyzed cascade reactions in total synthesis of natural product. Tetrahedron Lett. 58, 4420-4426. doi: 10.1016/j.tetlet.2017.10.029

Taber, D. F., Raman, K., and Gaul, M. D. (1987). Enantioselective ring construction: synthesis of (+)-estrone methyl ether. J. Org. Chem. 52, 28-34. doi: 10.1021/jo00377a005

Tian, Y., Tian, L., He, X., Li, C.-J., Jia, X., and Li, J. (2015). Indium (III) chloridecatalyzed isocyanide insertion reaction to construct complex spirooxindole. Org. Lett. 17, 4874-4877. doi: 10.1021/acs.orglett.5b02432
Tietze, L. F., Kinzel, T., and Brazel, C. C. (2009). The domino multicomponent allylation reaction for the stereoselective synthesis of homoallylic alcohols. Acc. Chem. Res. 42, 367-378. doi: 10.1021/ar800170y

Toda, F., Tanaka, K., Sano, I., and Isozaki, T. (1994). A new synthetic route to 1, 2-dihydrocyclobutaarenes. Angew. Chem. Int. Ed. 33, 1757-1758. doi: $10.1002 /$ anie. 199417571

Toda, M., Okada, K., and Oda, M. (1988). Synthesis of 3, 4; 7, 8; 9, 10tribenzobicyclo [4.2.2] deca-1,3,7,9-tetraene: a new strained bridgehead olefin. Tetrahedron Lett. 29, 2329-2332. doi: 10.1016/S0040-4039(00)86051-7

Trost, B. M. (1995). Atom economy - a challenge for organic synthesis: homogeneous catalysis leads the way. Angew. Chem. Int. Ed. 34, 259-281. doi: $10.1002 /$ anie. 199502591

Trost, B. M. (2002). On inventing reactions for atom economy. Acc. Chem. Res. 35, 695-705 doi: $10.1021 / \operatorname{ar} 010068 \mathrm{z}$

Trost, B. M., Toste, F. D., and Greenman, K. (2003). Atom economy. palladiumcatalyzed formation of coumarins by addition of phenols and alkynoates via a Net C-H insertion. J. Am. Chem. Soc. 125, 4518-4526. doi: 10.1021/ja02 86573

Vlaar, T., Maes, B.-U., Ruijter, E., and Orru, R.-V. (2013). Palladium-catalyzed migratory insertion of isocyanides: an emerging platform in cross-coupling chemistry. Angew. Chem. Int. Ed. 52, 7084-7097. doi: 10.1002/anie.2013 00942

Wang, J.-Y., Xie, F.-L., Hu, J.-Q., Yang, S.-Z., Wang, Y.-J., Hao, W.-J., et al. (2018). Atom-economic synthesis of cyclobuta[a]naphthalen-4-ols via base-promoted $[2+2]$ cycloaddition/1,6-nucleophilic addition cascades. Org. Biomol. Chem. 16, 7104-7108. doi: 10.1039/C8OB02042F

Wang, J.-Y., Zhou, P., Li, G., Hao, W.-J., Tu, S.-J., and Jiang, B. (2017). Synthesis of functionalized benzo $[g]$ indoles and 1-naphthols via carboncarbon triple bond breaking/rearranging. Org. Lett. 19, 6682-6685. doi: 10.1021/acs.orglett.7b03410

Wang, L., Shi, L.-X., Liu, L., Li, Z.-X., Xu, T., Hao, W.-J., et al. (2017). Synthesis of diastereoenriched oxazolo[5,4-b]indoles via catalyst-free multicomponent bicyclizations. J. Org. Chem. 82, 3605-3611. doi: 10.1021/acs.joc. 7b00129

Wang, Y., Lu, H., and Xu, P.-F. (2015). Asymmetric catalytic cascade reactions for constructing diverse scaffolds and complex molecules. Acc. Chem. Res. 48, 1832-1844. doi: 10.1021/acs.accounts.5b00217

Warrener, R. N., Pitt, I. G., and Russell, R. A. (1993). Reaction of dienophiles with a 5, 6-bismethylenenorborn-2-en-7-one: the synthesis of a stable norborna-2, 5-dien-7-one. Aust. J. Chem. 46, 1009-1019. doi: 10.1071/CH99 31009

Wei, H., Zhai, H., and Xu, P.-F. (2009). Novel platinum-catalyzed tandem reaction: an efficient approach to construct naphtho[1,2-b]furan. J. Org. Chem. 74, 2224-2226. doi: 10.1021/jo802645s

Zhang, C., Hooper, J. F., and Lupton, D. W. (2017). N-heterocyclic carbene catalysis via the $\alpha, \beta$-unsaturated acyl azolium. ACS Catal. 7, 2583-2596. doi: $10.1021 /$ acscatal.6b03663

Conflict of Interest Statement: The authors declare that the research was conducted in the absence of any commercial or financial relationships that could be construed as a potential conflict of interest.

Copyright $\odot 2018 \mathrm{Li}$, Hao, Li, Tu and Jiang. This is an open-access article distributed under the terms of the Creative Commons Attribution License (CC BY). The use, distribution or reproduction in other forums is permitted, provided the original author(s) and the copyright owner(s) are credited and that the original publication in this journal is cited, in accordance with accepted academic practice. No use, distribution or reproduction is permitted which does not comply with these terms. 